Revue des patrimoines

$24 \mid 2014$

Architecture et urbanisme de villégiature : un état de la recherche

\title{
Aix-les-Bains, carrefour de villégiatures : thermalisme, climatisme, sports d'hiver et bords de lac
}

\section{Elsa Belle}

\section{(2) OpenEdition}

\section{Journals}

Édition électronique

URL : http://journals.openedition.org/insitu/11132

DOI : 10.4000/insitu. 11132

ISSN : 1630-7305

Éditeur

Ministère de la Culture

Référence électronique

Elsa Belle, «Aix-les-Bains, carrefour de villégiatures : thermalisme, climatisme, sports d'hiver et bords de lac », In Situ [En ligne], 24 | 2014, mis en ligne le 21 juillet 2014, consulté le 07 juillet 2020. URL : http://journals.openedition.org/insitu/11132 ; DOI : https://doi.org/10.4000/insitu.11132

Ce document a été généré automatiquement le 7 juillet 2020.

\section{(i) $(9)$}

In Situ Revues des patrimoines est mis à disposition selon les termes de la licence Creative Commons Attribution - Pas d'Utilisation Commerciale - Pas de Modification 4.0 International. 


\section{Aix-les-Bains, carrefour de villégiatures : thermalisme, climatisme, sports d'hiver et bords de lac}

\section{Elsa Belle}

1 Baignée par les eaux du lac du Bourget, la ville thermale d'Aix-les-Bains, symbolisée sur cette image (fig. 1) par son parc thermal occupant généreusement l'espace urbain, s'étire au pied du Revard, accessible grâce au chemin de fer à crémaillère vanté par cette réclame ${ }^{1}$. C'est donc l'idée d'un lieu situé à la confluence des eaux thermales, de la montagne et de l'eau douce, que popularise cette affiche de la Compagnie des chemins de fer Paris-Lyon-Méditerranée à la fin du XIX ${ }^{\mathrm{e}}$ siècle. 
Figure 1

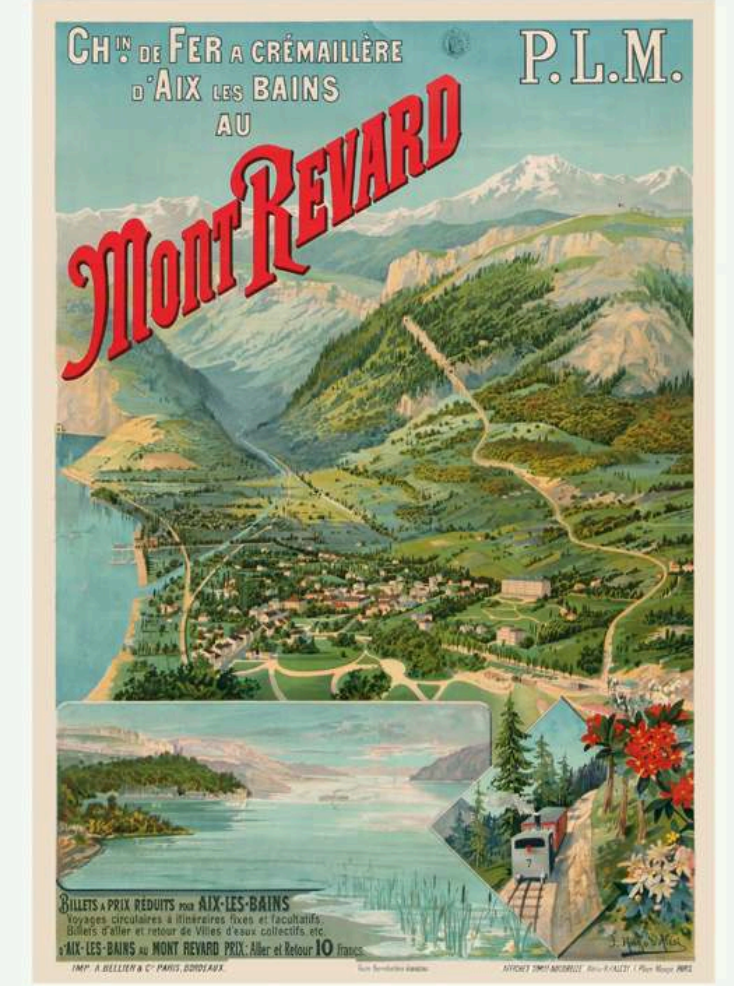

Publicité de la Compagnie PLM pour le Mont-Revard, fin du xix ${ }^{\mathrm{e}}$ siècle. Reproduction d'une affiche du PLM, avec l'aimable autorisation de Wagons-Lits Diffusion. Droits réservés.

2 Située en Savoie, la ville d'Aix-les-Bains, implantée sur des sources thermales, est bordée à l'ouest par le lac du Bourget, plus grand lac naturel de France après le lac Léman, et se développe à l'est sur les balcons du massif des Bauges couronné par le mont Revard culminant à 1545 mètres d'altitude. La topographie de la partie est de la ville, comprenant le centre historique, est ainsi marquée par une importante déclivité est-ouest, tandis que celle de la partie ouest se caractérise par un terrain relativement plat (fig. 2, 3). Avec 30000 curistes accueillis en 2012, Aix-les-Bains est actuellement la troisième station thermale de France, spécialisée dans le traitement des rhumatismes et des maladies oto-rhino-laryngologiques. Elle bénéficie du label France station nautique accordée à trente-six villes en France. Le Revard, relié à La Féclaz, constitue aujourd'hui le plus grand domaine de ski nordique de France ${ }^{2}$. 
Figure 2

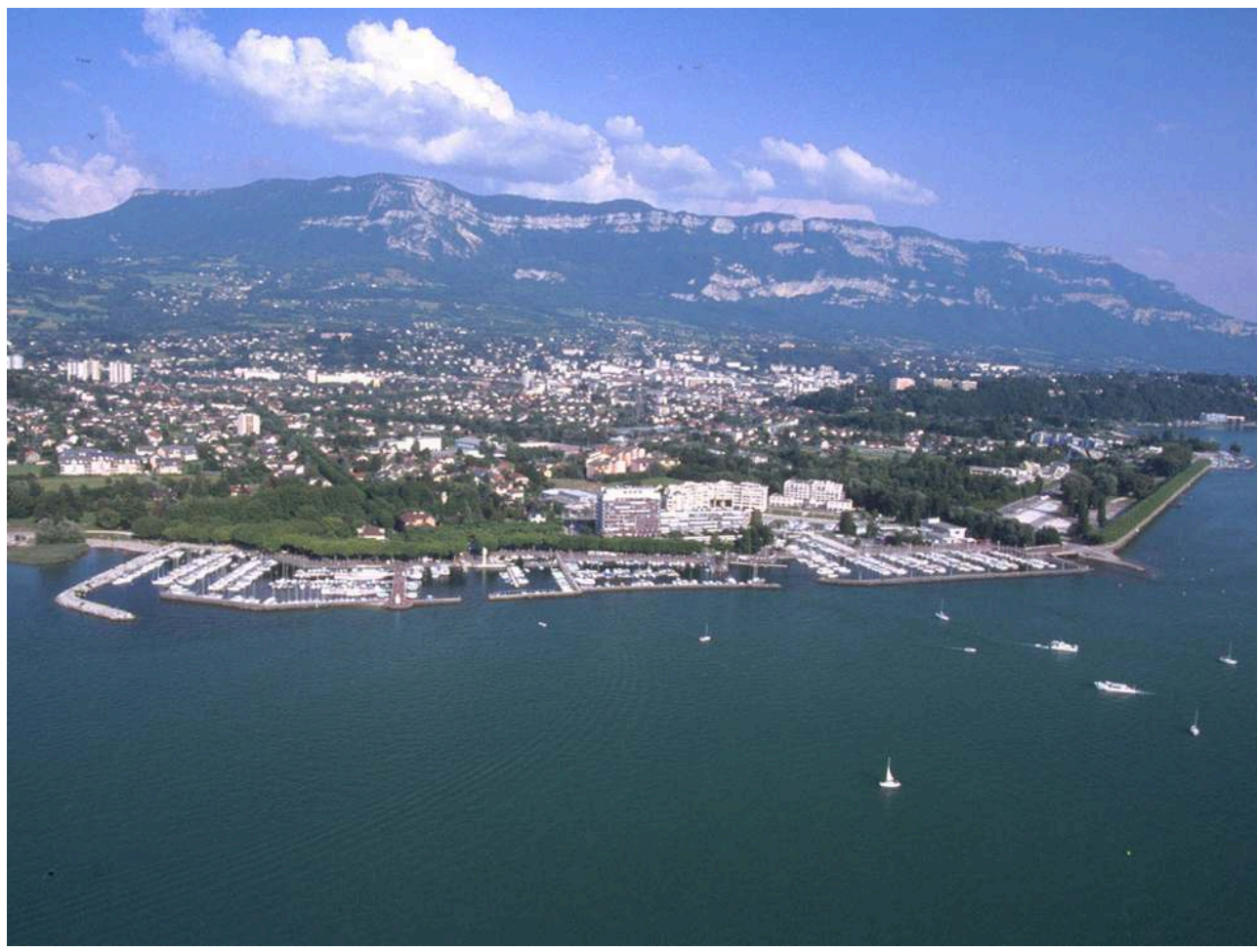

Aix-les-Bains, entre lac du Bourget et massif des Bauges.

(C) Archives municipales d'Aix-les-Bains.

Figure 3

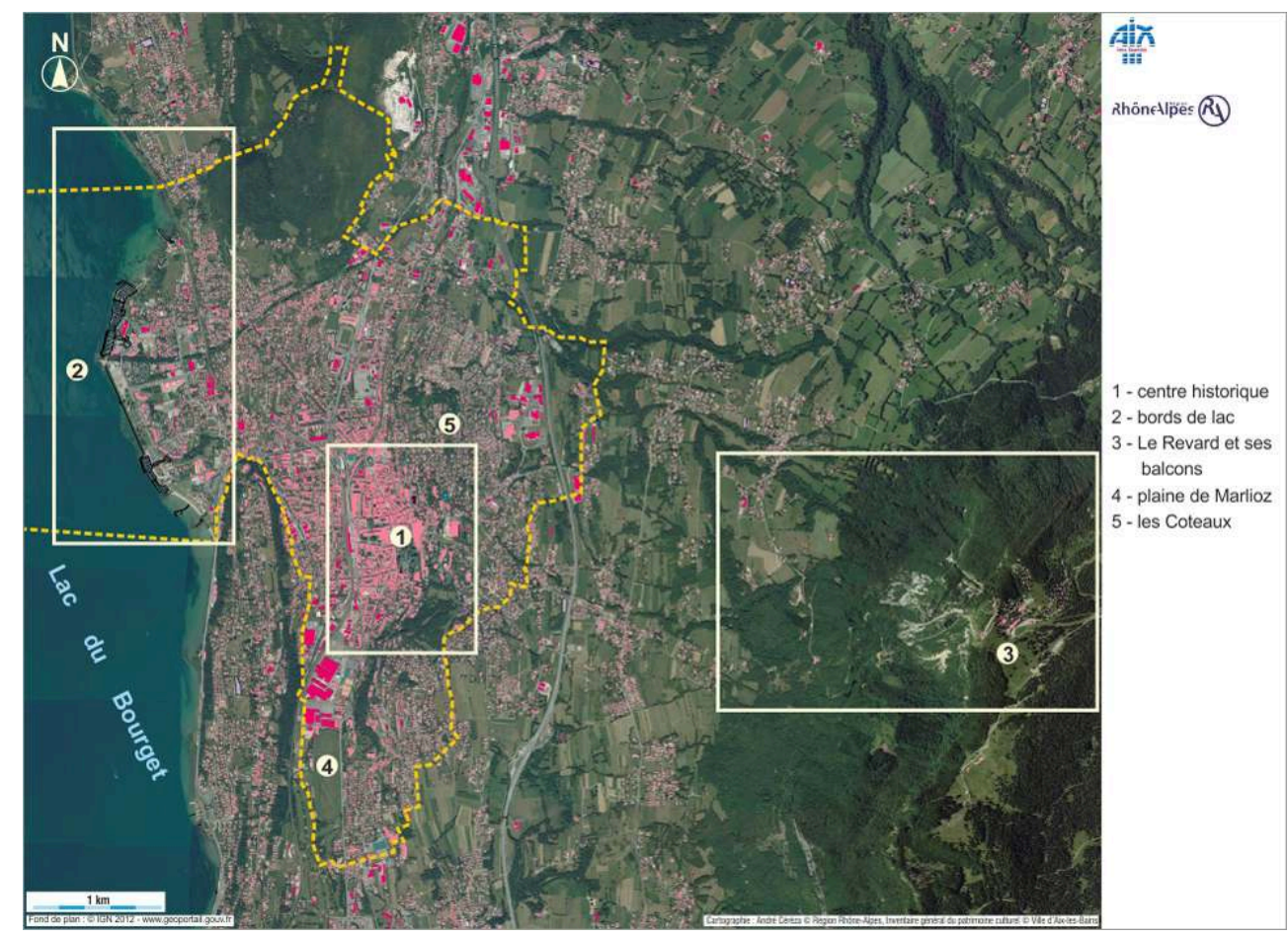

Carte générale de localisation.

Cartog. Céréza, André, 2014. @ Région Rhône-Alpes, service de l'Inventaire général du patrimoine culturel. (c) Ville d'Aix-les-Bains. 
3 L'intérêt pour les eaux thermales, pour la montagne et pour les bords de lac, ainsi que la mise en valeur de ces ressources, ont cependant été rythmés par des chronologies différentes. Les formes de villégiature qui y sont liées n'ont donc pas été déclinées avec la même importance dans l'architecture et l'urbanisme aixois. Depuis la fin du XVIII ${ }^{\mathrm{e}}$ siècle, c'est le thermalisme qui marque sans conteste le plus fortement le territoire dans ses dimensions économiques, sociales, urbaines et architecturales. Sous son impulsion, climatisme, sports d'hiver et activités balnéaires connaissent un développement convergent dans la première moitié $\mathrm{du} \mathrm{xx}^{\mathrm{e}}$ siècle.

4 À partir du début $d u x x^{e}$ siècle, la station thermale aixoise se mue progressivement en véritable ville d'eaux. Qualifiée par ailleurs de «station mondaine » à partir de 1880 , elle est fréquentée par "des baigneurs et des étrangers». Ces deux formules, employées dans les documents de l'époque ${ }^{3}$, permettent de mieux cerner l'ambiance des lieux. L'expression "baigneurs et étrangers " peut être assimilée à ceux que l'on nomme aujourd'hui les curistes et les touristes, et désignait jusqu'au milieu du $\mathrm{xx}^{\mathrm{e}}$ siècle les personnes suivant une cure, accompagnées de leur entourage ainsi que les simples villégiateurs ${ }^{4}$. Les uns comme les autres étaient animés en outre par le désir de voir et de se faire voir dans la "station mondaine ». À côté des contraintes imposées par la cure et des agréments offerts par la ville de plaisirs, ceux-ci visitent les espaces naturels environnant Aix. En 1816, c'est en la sauvant d'une noyade dans le lac du Bourget que Lamartine rencontre Julie Charles, alias Elvire ${ }^{5}$. La popularité de ces lieux, encouragée par le développement du romantisme et du goût pour le pittoresque, est assurée par leur promotion dans les guides touristiques édités tout au long du XIX siècle. De l'intérêt des "baigneurs et des étrangers ", relayé par les guides, naît un attrait croissant chez les élites politiques et économiques de la ville, dominées par les médecins et les hôteliers, pour ces espaces dont l'aménagement et la mise en valeur sont alors perçus comme des moyens permettant le développement de la ville et le maintien de son rang dans un contexte concurrentiel entre stations mondaines. Si les premiers aménagements du Revard et des bords de lac sont donc directement liés au thermalisme, le développement de ces espaces acquiert cependant progressivement une logique propre et autonome.

\section{Le développement de la ville autour du thermalisme}

5 Par sa longévité, par l'ampleur de son développement, par ses évolutions et par ses promoteurs, le thermalisme a modelé l'économie, la sociologie, l'urbanisme et l'architecture de la ville. Retracer l'histoire de l'établissement thermal permet de dessiner les principales évolutions de la station et de mettre en lumière ses singularités.

6 La première particularité réside dans l'existence de bains romains, attestée par la présence de vestiges situés aujourd'hui au cœur de l'établissement thermal (voir fig. 4). En effet, et cela constitue une autre singularité du thermalisme aixois, le bâtiment des thermes, construit à la fin du XVIII siècle, agrandi et modifié au cours des $\mathrm{XIX}^{\mathrm{e}}$ et $\mathrm{Xx}^{\mathrm{e}}$ siècles, a bénéficié jusqu'à récemment d'une implantation séculaire dictée notamment par la proximité des griffons, c'est-à-dire des points d'émergence des sources d'eau thermale. Les principales phases de développement de la ville peuvent se lire à travers les grandes campagnes d'agrandissement de l'établissement thermal 
puisque chacune d'entre elles a entraîné des aménagements urbains ainsi que la construction de lieux dédiés à la villégiature (fig. 4).

Figure 4

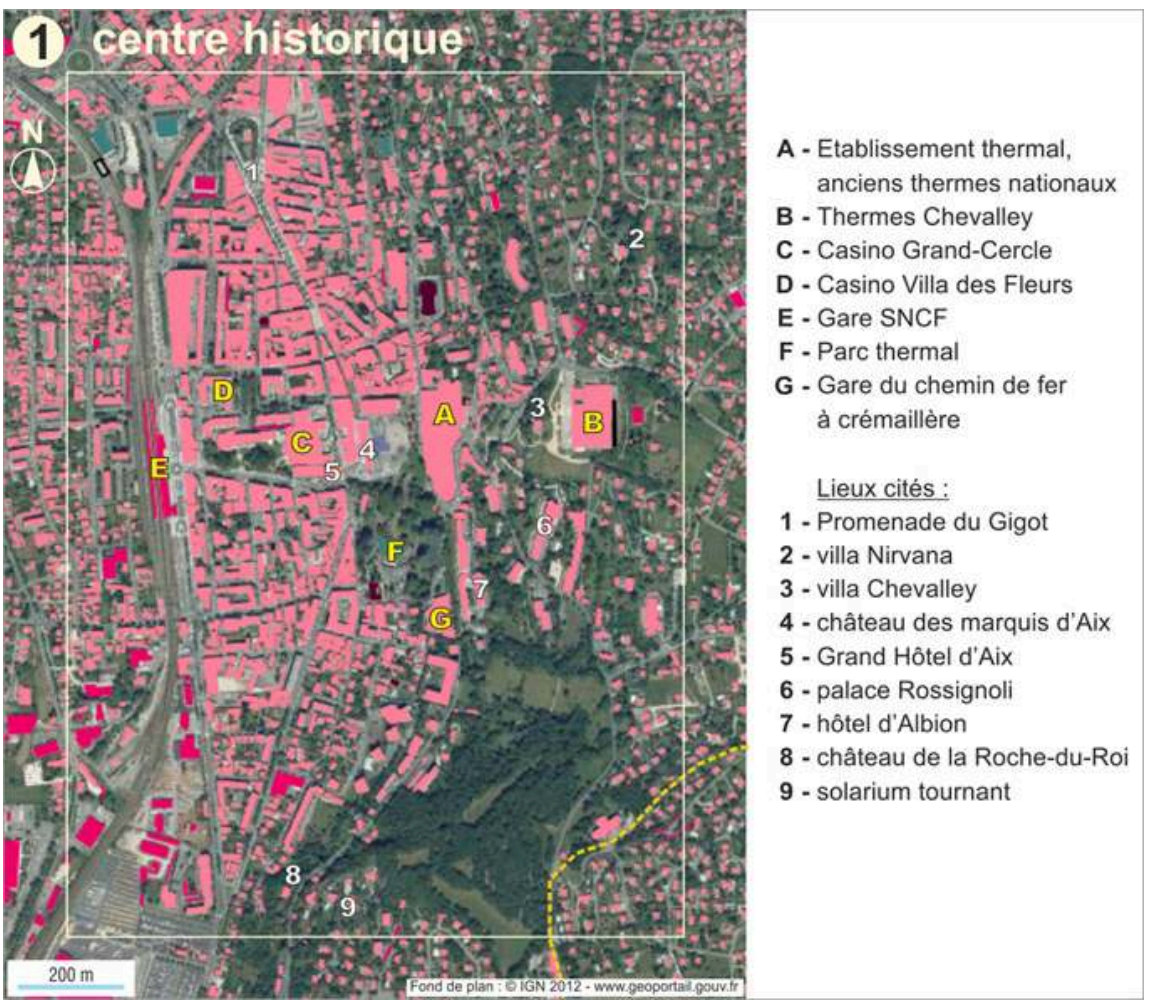

Carte de localisation, le centre-ville.

Cartog. Céréza, André, 2014. @ Région Rhône-Alpes, service de l'Inventaire général du patrimoine culturel. (c) Ville d'Aix-les-Bains.

\section{Le démarrage du thermalisme : la construction du Bâtiment royal des Bains}

7 La construction du Bâtiment royal des Bains en 1783, à l'initiative du roi Victor-Amédée III, signe le redémarrage du thermalisme aixois, tombé en sommeil depuis le $\mathrm{IV}^{\mathrm{e}}$ siècle. Ce bâtiment d'architecture classique se compose de deux ailes en saillie encadrant un corps central marqué d'un ordre colossal couronné d'un fronton (fig. 5). 


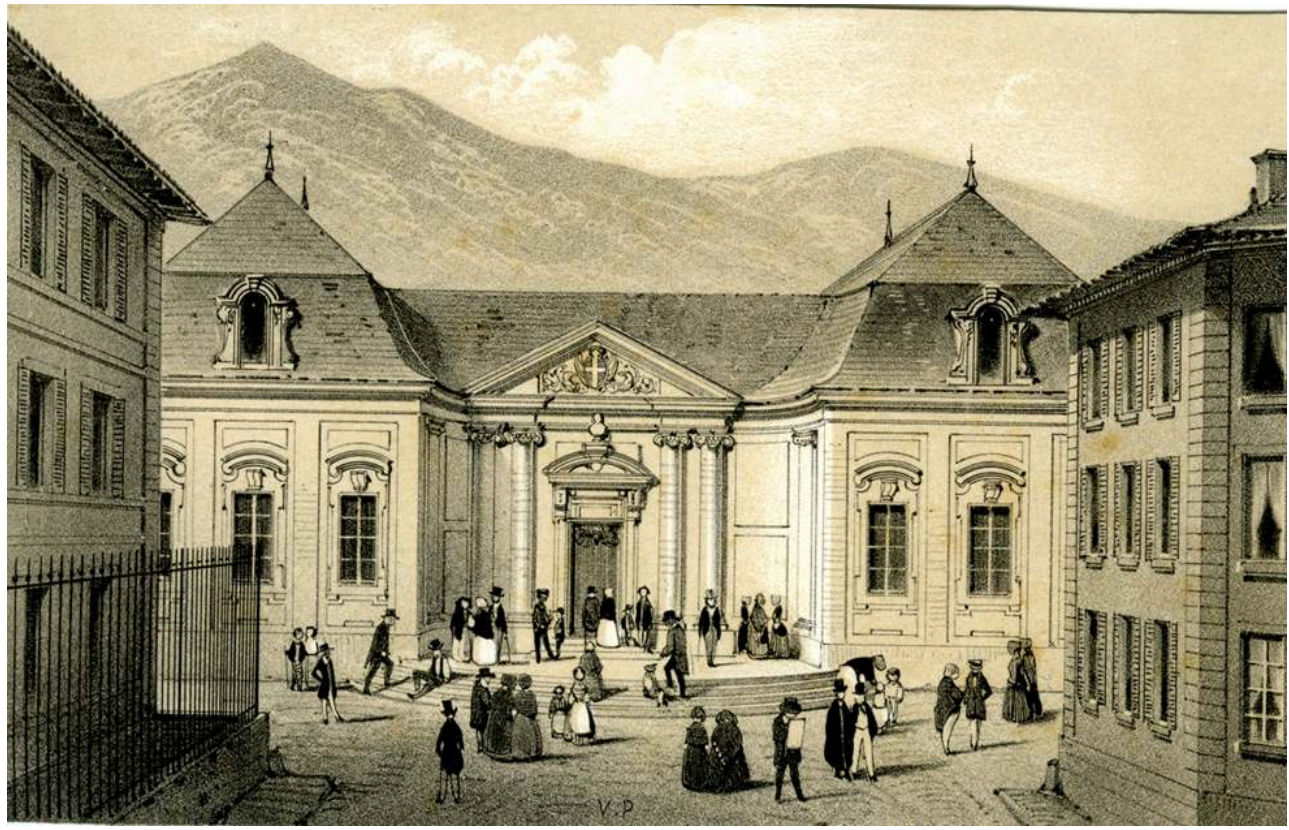

Le Bâtiment royal des Bains, milieu du xıx siècle.

(c) Archives municipales d'Aix-les-Bains.

8 À la suite de l'édification du bâtiment thermal, la Ville crée une promenade pour les villégiateurs, baptisée promenade du Gigot, adoptant la forme d'une fourche et située en dehors des murs de la cité (voir fig. 4). Devenue aujourd'hui la rue de Genève, cette artère constitue la principale rue commerçante de la ville et un axe de circulation important.

9 La première moitié du XIX siècle voit l'ouverture de plusieurs pensions et hôtels, installés dans des bâtiments préexistants, pour accueillir baigneurs et étrangers ${ }^{6}$. À l'instar des pensions Perrier et Bocquin, disparues, les témoignages de ces établissements ne sont cependant conservés bien souvent que dans les archives. À côté de ces modestes équipements, d'autres formes d'hébergement pour un autre type de clientèle voient le jour. Le début du XIX ${ }^{e}$ siècle est en effet également marqué par les premières villégiatures aristocratiques. La villa Chevalley, implantée au-dessus et à quelques encablures de l'établissement thermal, a accueilli les séjours des membres de la famille impériale napoléonide puis ceux de la famille royale sarde (voir fig. 4). Cette villa datant de la fin $d u \mathrm{XVII}^{\mathrm{e}}$ siècle était également appréciée pour ses jardins, souvent cités dans les guides touristiques jusqu’à la fin du xix siècle (fig. 6). 
Figure 6

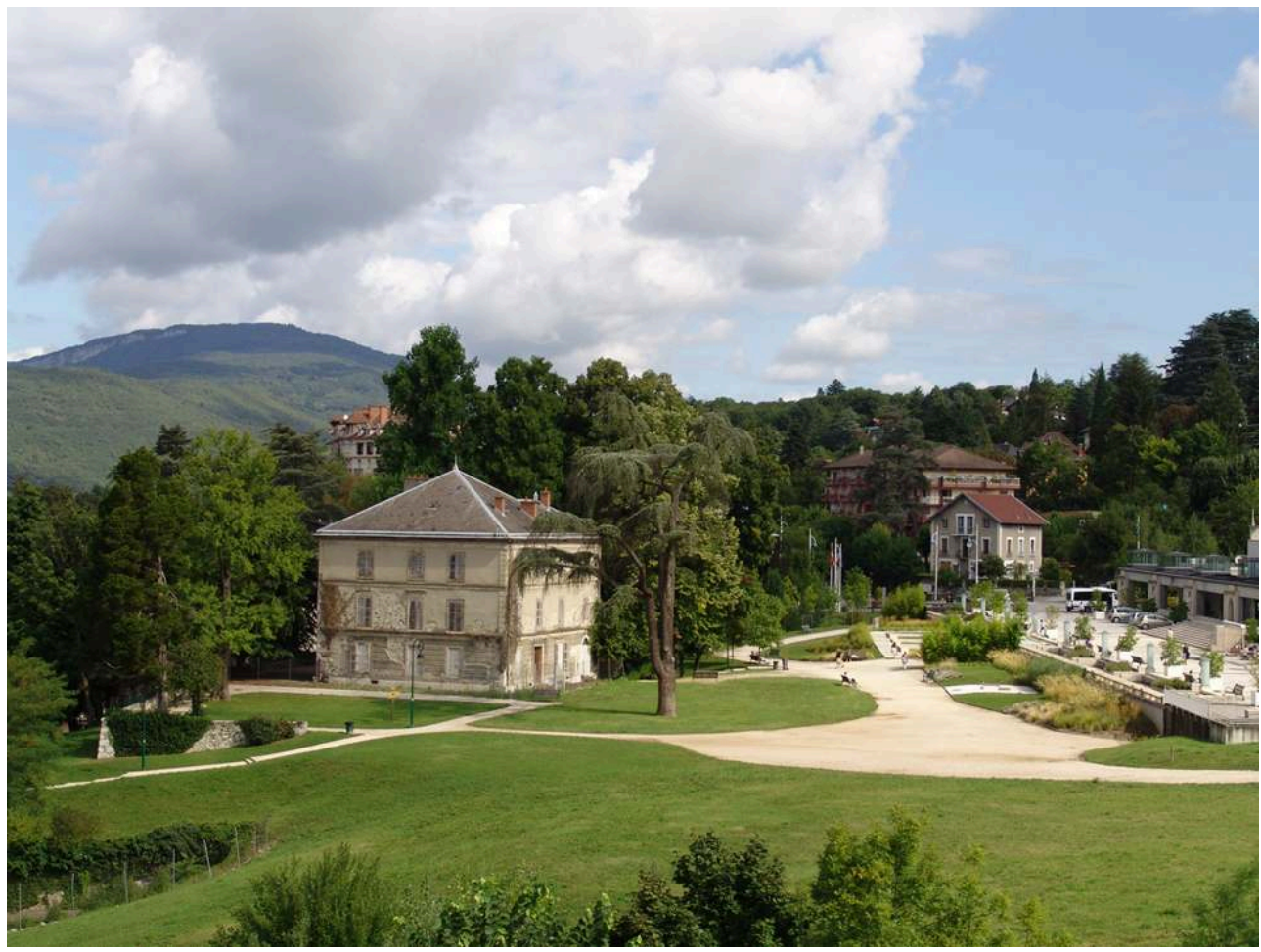

La villa Chevalley et son parc

Phot. Fouger, François, 2012. @ Région Rhône-Alpes, service de l'Inventaire général du patrimoine culturel. (c) Ville d'Aix-les-Bains.

Complément de la ville thermale, la ville de plaisirs émerge en 1824 avec la création d'un cercle, soit d'un lieu de rencontres, d'échanges et de jeux dédié aux villégiateurs. Installé d'abord dans le château des marquis d'Aix, le cercle commandite la construction d'un casino en 1849 à l'architecte Charles Pellegrini. Bien qu'implanté à l'extérieur des remparts de la ville, le casino Grand-Cercle se situe à proximité du Bâtiment royal des Bains (voir fig. 4). L'établissement est toujours en activité et le bâtiment qui l'abrite a fait l'objet de plusieurs campagnes d'agrandissement et d'embellissement jusqu'à récemment (fig. 7).

Figure 7

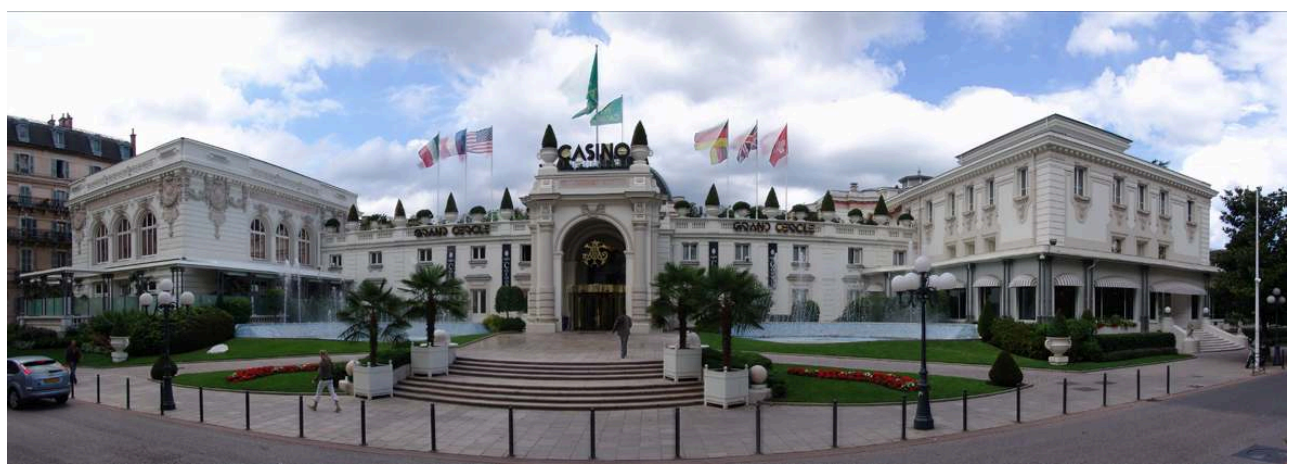

Le casino Grand-Cercle.

Phot. Fouger, François, 2009. (C Région Rhône-Alpes, service de l'Inventaire général du patrimoine culturel. (c) Ville d'Aix-les-Bains. 


\section{L'impulsion donnée au thermalisme par Napoléon III : l'achèvement des thermes Pellegrini}

11 Dans les années 1850, l'établissement thermal fait l'objet d'un projet d'agrandissement important mené par l'architecte du casino Grand-Cercle, Charles Pellegrini. Mais, faute d'argent, le chantier est arrêté en 1858. La visite de Napoléon III en Savoie en 1860, suite au rattachement de l'ancien duché à la France, s'avère décisive puisque l'empereur accepte de financer l'achèvement $d u$ chantier en échange de la nationalisation des sources et de l'établissement thermal. Les thermes Pellegrini prennent place devant le Bâtiment royal des Bains dont ils masquent désormais la façade principale ${ }^{7}$. Leur présence sur la rue se caractérise par une élévation néoclassique, rythmée de trois grandes baies en plein cintre donnant accès au hall d'accueil central (fig. 8).

Figure 8

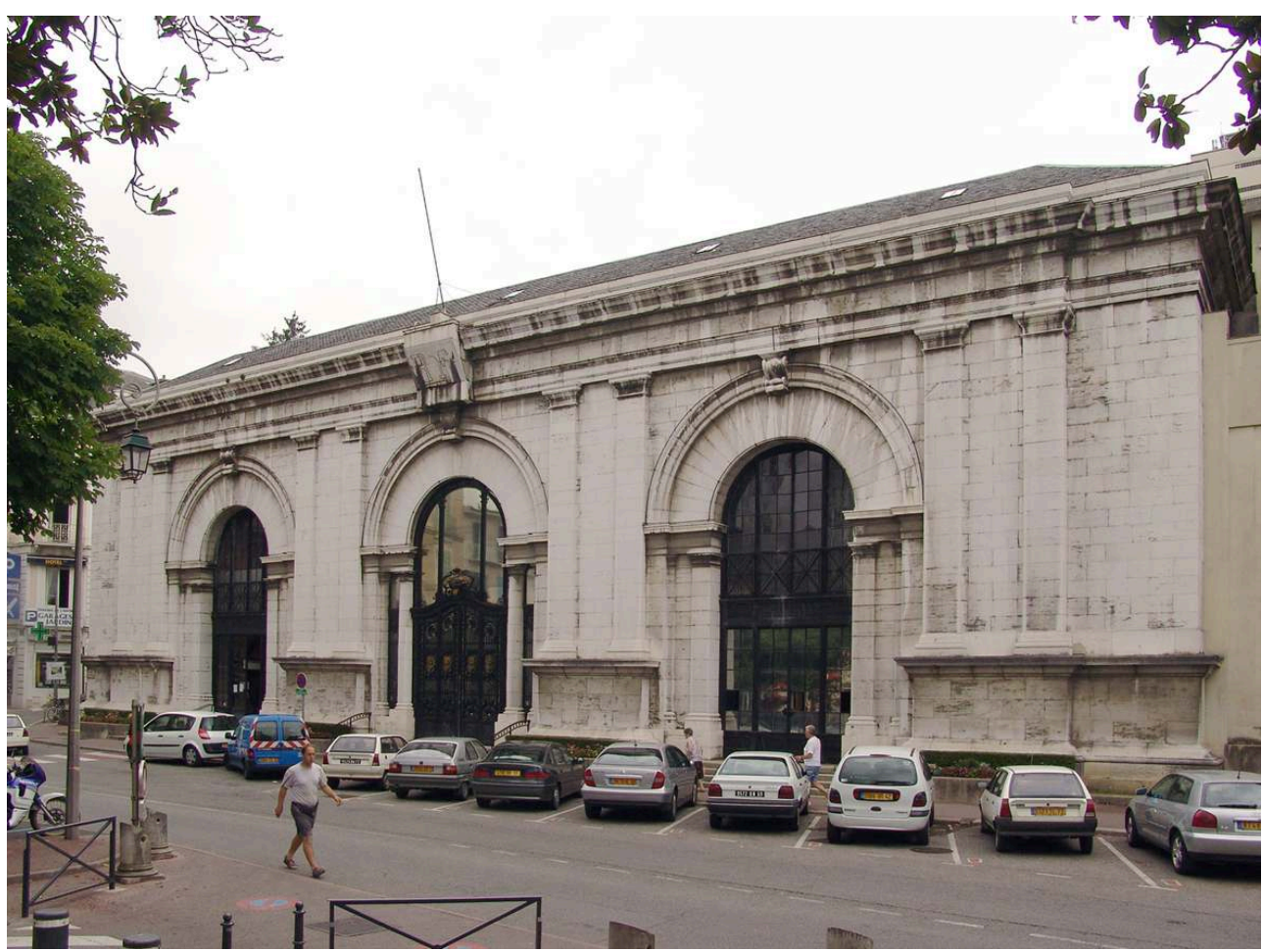

La façade des thermes Pellegrini.

Phot. Fouger, François, 2004. @ Région Rhône-Alpes, service de l'Inventaire général du patrimoine culturel. (c) Ville d'Aix-les-Bains.

12 Le rattachement de la Savoie à la France, la nouvelle ampleur donnée à l'établissement de bains, l'arrivée du chemin de fer à Aix et la construction d'une gare à proximité du centre-ville (voir fig. 4) donnent une impulsion décisive au développement de la ville. Une véritable fièvre constructive s'empare alors de la station, se traduisant par l'aménagement de nouveaux équipements et l'érection d'hôtels, de palaces et de villas rivalisant d'ostentation et de luxe.

13 L'État, désormais propriétaire des thermes, et le conseil municipal, constatant l'absence d'un lieu de promenade dédié aux baigneurs, décident la création d'un parc thermal en 
1865 dans le clos du château des marquis d'Aix (voir fig. 4). Fréquenté dès le deuxième quart du XIX ${ }^{e}$ siècle par les villégiateurs du fait de sa proximité avec les thermes et avec le château investi par le cercle à partir de 1824, ce lieu mondain semblait tout désigné. L'architecte Samuel Revel, chargé d'élaborer le dessin du parc, propose celui d'un jardin anglais propice à la déambulation.

14 À l'inverse, malgré la présence d'un casino, Alphonse Mottet crée un second établissement de spectacles et de jeux appelé la Villa des Fleurs, inauguré en 1879 (voir fig. 4). Incendié dans les années 1950 puis démoli, ce casino était logé dans une villa existante progressivement transformée et embellie entre 1880 et 1910 pour accueillir notamment un théâtre, une salle de baccarat, une scène de music-hall, un restaurant, une salle des petits chevaux et des salons de lecture. À la fin du xixe siècle, la concurrence entre les deux casinos Grand-Cercle et Villa des Fleurs, d'autant plus manifeste que les établissements étaient voisins, était perçue par l'historien Mouxy de Loche comme le creuset d'une saine émulation.

La rivalité de ces deux Cercles, qui se touchent et offrent au public les mêmes attraits, les mêmes plaisirs, n'a pas eu jusqu'à présent d'inconvénients, au contraire; cet assaut pour faire mieux que son voisin, cette concurrence, en quelque sorte, a beaucoup contribué à la prospérité d'Aix'.

Alors que les pensions et les hôtels de la première moitié $\mathrm{du}$ XIX ${ }^{\mathrm{e}}$ siècle avaient investi des bâtiments préexistants, les établissements ouverts dans la seconde partie du siècle donnent lieu à de nouvelles constructions. Les premiers grands hôtels sont bâtis au centre-ville. Le Grand Hôtel d'Aix, achevé en 1857, s'installe entre le casino GrandCercle et les thermes (voir fig. 4). Premier grand hôtel édifié dans la ville, il s'agit également du premier hôtel à atrium à Aix-les-Bains ${ }^{9}$ (fig. 9). L'architecture intérieure d'un hôtel à atrium s'articule autour d'un premier niveau dédié aux espaces d'accueil et d'étages occupés par des chambres donnant sur un atrium central éclairé d'une verrière zénithale. 
Figure 9

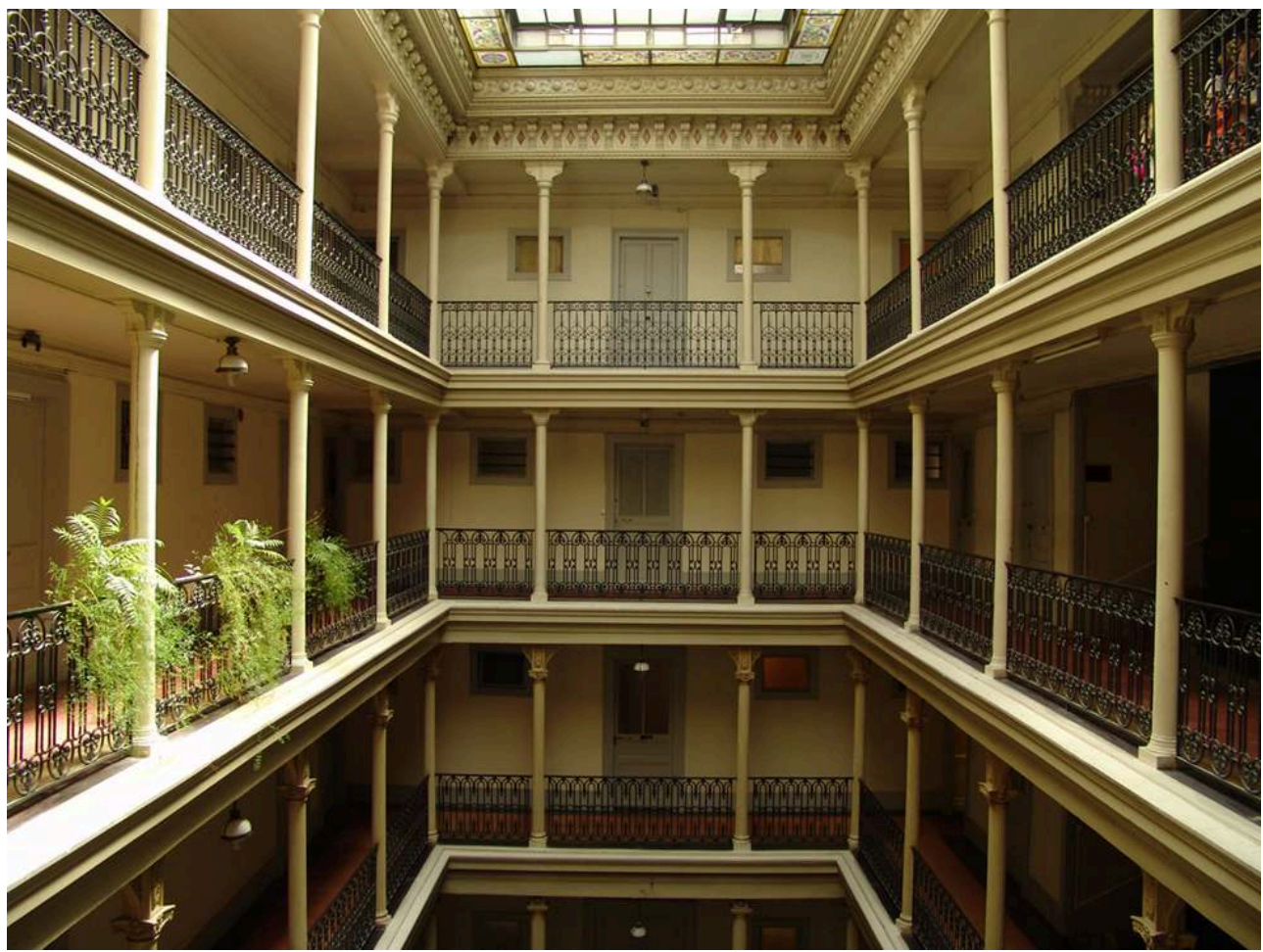

L'atrium du Grand Hôtel d'Aix.

Phot. Fouger, François, 2007. @ Région Rhône-Alpes, service de l'Inventaire général du patrimoine culturel. (c) Ville d'Aix-les-Bains.

16 À la fin du XIX ${ }^{e}$ siècle, villas et palaces investissent les points hauts de la ville situés à l'ouest, de manière à jouir de l'ensoleillement et du paysage donnant sur la colline de Tresserve, le lac et le massif de l'Épine. Commandités par Gaudens Rossignoli, les trois palaces Rossignoli sont bâtis entre 1884 et 1914 sur les hauteurs de la ville (voir fig. 4). Bien que les programmes soient identiques, les deux architectes suisses Antoine Gouy et Alfred Olivet proposent en l'espace de vingt ans des architectures différentes pour chacun de ces trois hôtels. Splendide (1884), Excelsior (1906) et Royal (1914) ne se ressemblent pas (fig. 10). Sur les hauteurs de la ville, les palaces et les grands hôtels voisinent avec de nombreuses villas dont les plus impressionnantes se concentrent dans le secteur appelé les Coteaux. 
Figure 10

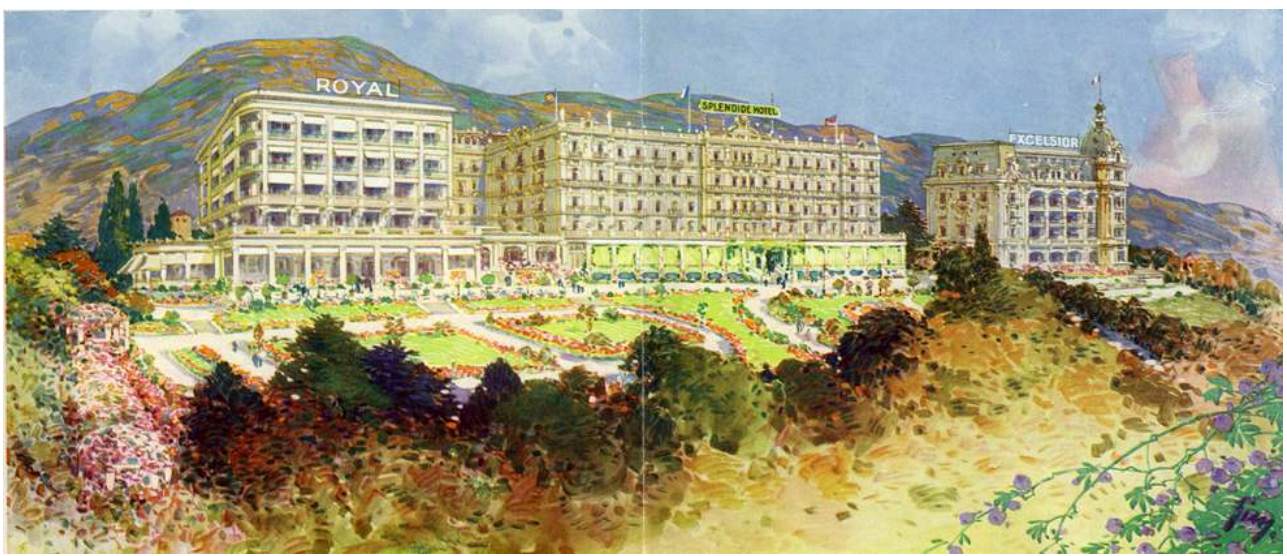

Les palaces Rossignoli, vers 1925.

(c) Archives municipales d'Aix-les-Bains.

La villa Nirvana, bâtie en 1897 pour Chapin Chester William, un Américain en villégiature à Aix, se situe sur l'un des points les plus hauts de la ville (voir fig. 4). Malgré cet emplacement privilégié, cette villa présente un belvédère permettant de jouir de la vue et officiant comme un signal (fig. 11).

Figure 11

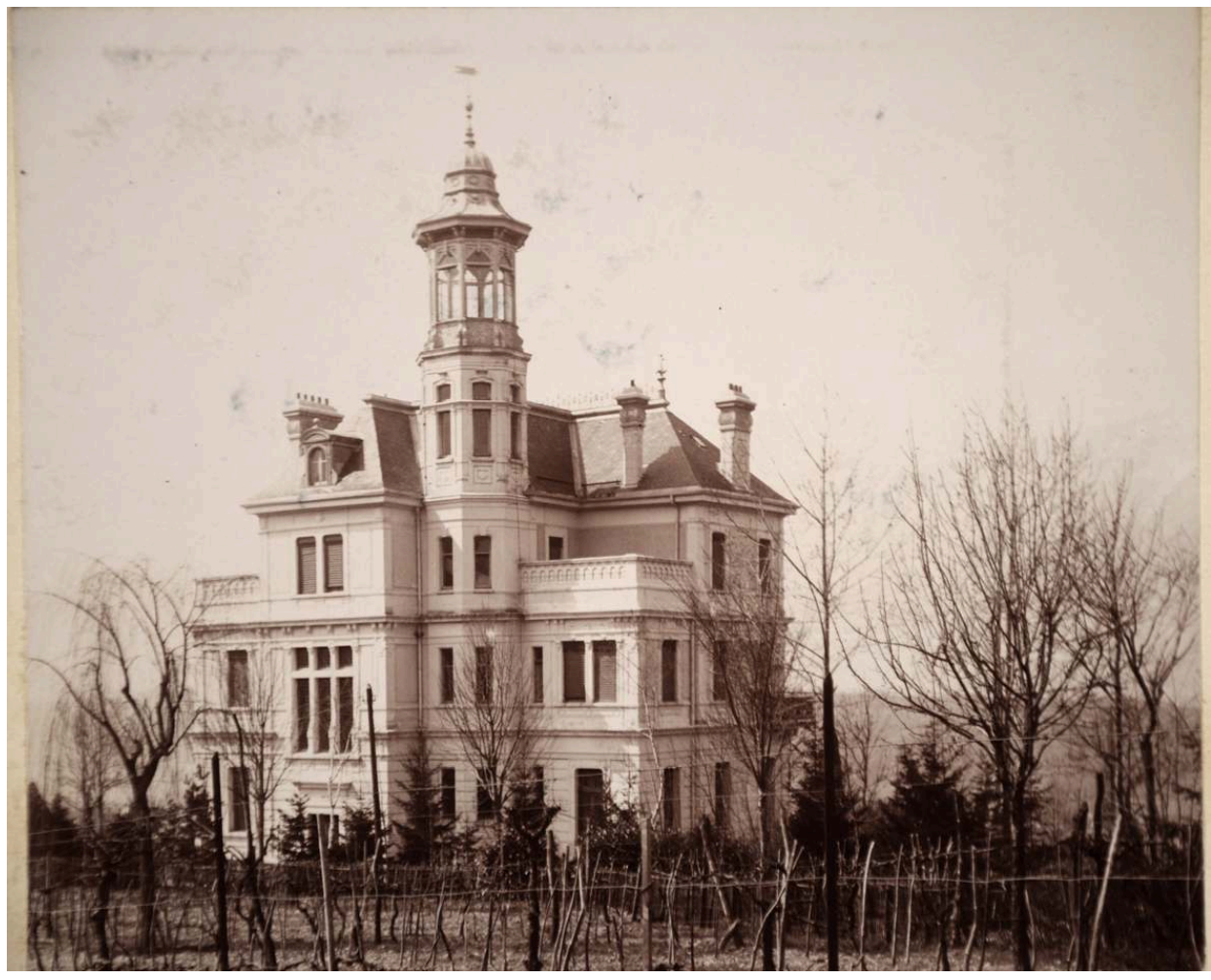

La villa Nirvana, vers 1900.

(c) Archives municipales d'Aix-les-Bains.

18 L'une des architectures les plus étonnantes de la ville reste pourtant le château de la Roche-du-Roi. Véritable folie, cette construction est dessinée par l'architecte Jules Pin pour Jean Archiprêtre, commanditaire de plusieurs villas implantées à proximité du 
château. L'édifice est achevé en 1900 après trois années de travaux. L'implantation du bâtiment, situé en hauteur sur une parcelle très pentue, a nécessité de très importants travaux de terrassement (voir fig. 4). Cette villa de plan rectangulaire cantonnée de tourelles circulaires coiffées de dômes adopte un style plus qu'éclectique (fig. 12). Visible depuis de nombreux lieux dans la ville, le château de la Roche-du-Roi a suscité la curiosité, comme l'attestent les nombreuses cartes postales qui le représentent.

Figure 12

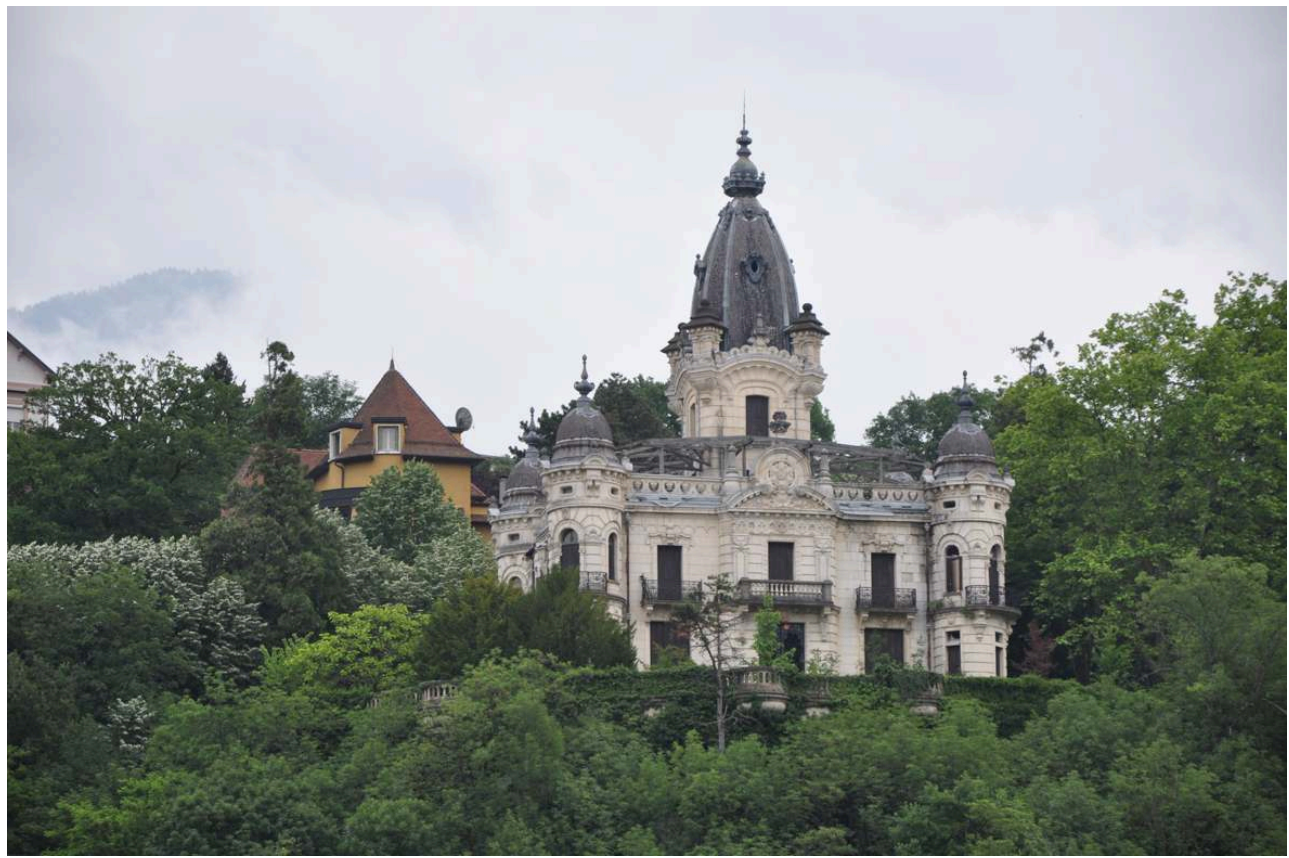

Le château de la Roche-du-Roi

Phot. Fouger, François, 2012. (C) Région Rhône-Alpes, service de l'Inventaire général du patrimoine culturel. (c) Ville d'Aix-les-Bains.

Le boulevard lui donnant accès constitue par ailleurs un bon exemple de l'évolution des promenades des Coteaux, investies par les villégiateurs pour la déambulation ou pour l'habitation et progressivement transformées en voies urbaines afin de desservir les nombreuses villas qui y ont été édifiées. L'appellation «boulevard " pour une voierie peu large, ainsi que des noms tels que «boulevard des Anglais » ou bien «boulevard de Paris ", sont choisies pour témoigner de l'aura de la station mondaine, qui connaît un véritable essor à partir de 1880 .

\section{Le grand chantier des années 1930 : les thermes Pétriaux}

Au lendemain de la Première Guerre mondiale, la direction des Thermes manifeste la volonté d'agrandir l'établissement thermal avec l'ambition d'en faire le plus luxueux d'Europe afin de relancer l'activité. Les travaux d'extension menés par l'architecte départemental Roger Pétriaux et achevés en 1933 se caractérisent ainsi par leur ampleur, par la modernisation des locaux et par le luxe qui y est déployé (fig. 13). La surface de l'établissement thermal est doublée et il est fait appel à des décorateurs de renom tels qu'Edgar Brandt pour la réalisation des ferronneries ou l'entreprise Gentil et Bourdet pour les céramiques ornant le hall d'entrée et les cabines de luxe (fig. 14). 
La construction des thermes Pétriaux marque un temps fort dans l'histoire de l'organisation de la ville et dans celle de l'architecture de l'établissement thermal. En effet, elle renforce la centralité du bâtiment, devenu un véritable monument, dans le tissu urbain, et lui donne sa physionomie actuelle même si son allure générale est modifiée dans les années 1970 à la suite du chantier conduit par l'architecte Claude Mabileau.

Figure 13

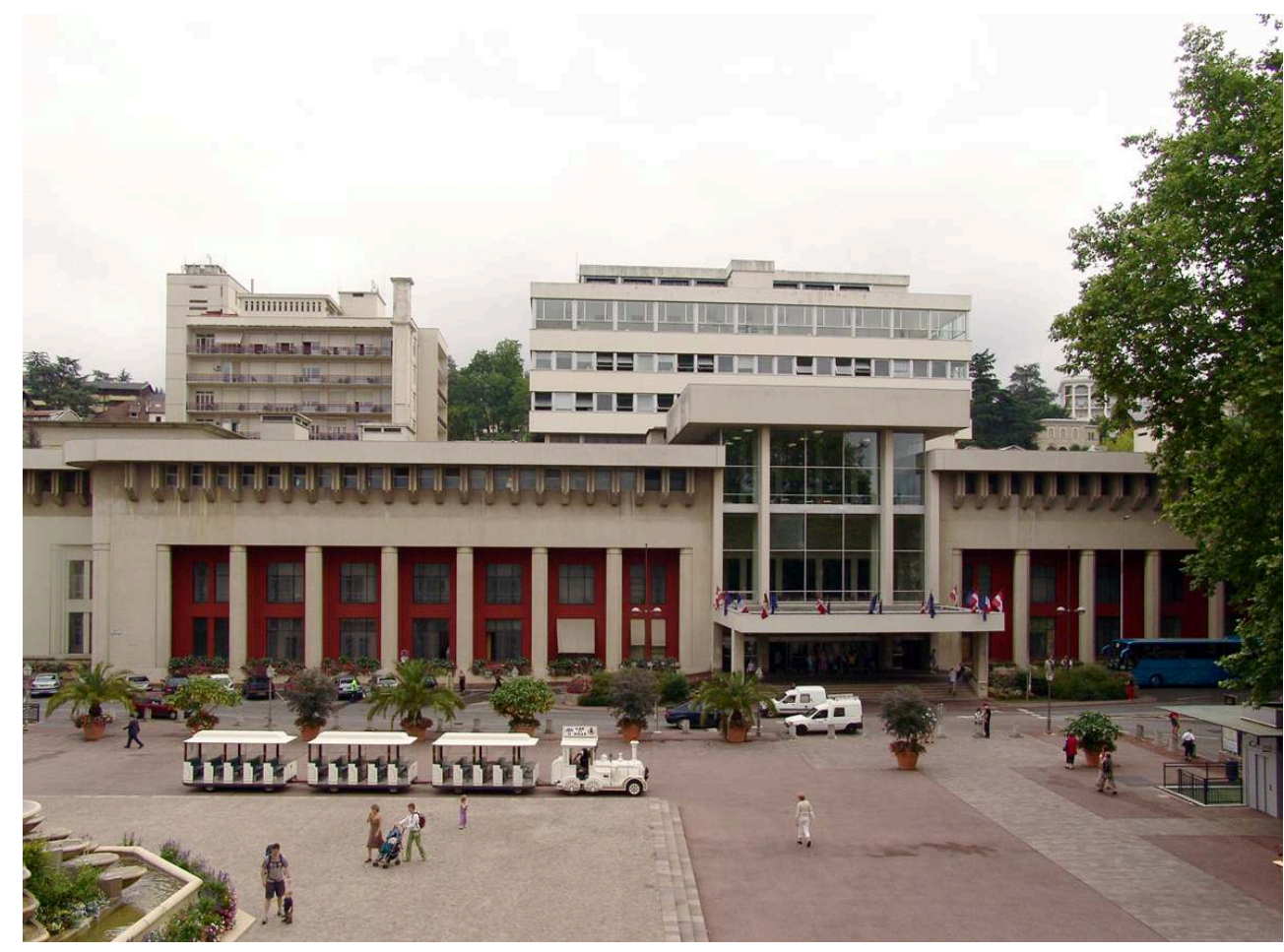

La façade des thermes Pétriaux, modifiée après les travaux menés par Claude Mabileau.

Phot. Fouger, François, 2004. (c) Région Rhône-Alpes, service de l'Inventaire général du patrimoine culturel. (c) Ville d'Aix-les-Bains. 
Figure 14

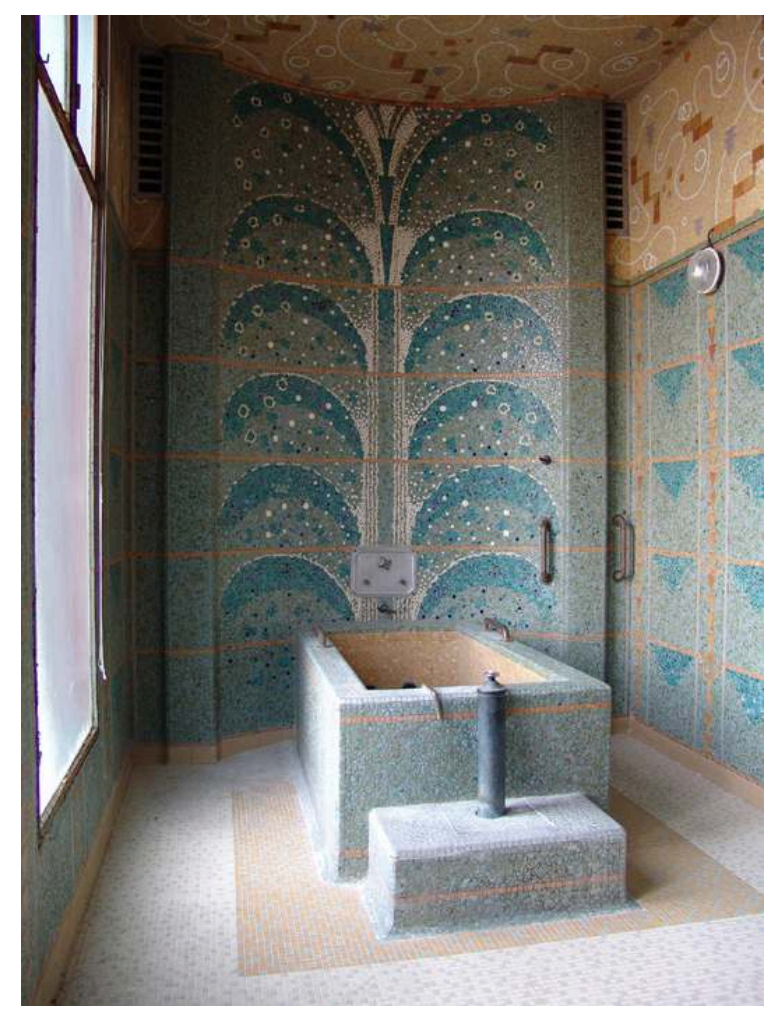

Une cabine de luxe des thermes Pétriaux.

Phot. Fouger, François, 2004. (c) Région Rhône-Alpes, service de l'Inventaire général du patrimoine culturel. (c) Ville d'Aix-les-Bains.

Les travaux engagés aux thermes entraînent le réaménagement complet du parc thermal. Tandis que son remaniement est confié à l'architecte paysagiste parisien Bouhana, différents équipements tels qu'un promenoir des sources, des pergolas et des kiosques sont réalisés par Roger Pétriaux. Ici, comme à l'établissement thermal, la patte de cet architecte qui se caractérise par l'usage d'un style Art déco très prononcé est immédiatement reconnaissable.

Bien que moins nombreuses et moins ambitieuses, les constructions de villas et d'hôtels se poursuivent à la suite de l'inauguration des thermes Pétriaux. Située sur les hauteurs et à quelques mètres du château de la Roche-du-Roi, la villa La Joie de Vivre constitue un très bel exemple de l'évolution de l'histoire du goût. Alors que les premiers plans présentés par l'architecte Jules Pin au commanditaire proposent une architecture très classique, ce dernier préfère recourir à un architecte niçois qui réalise une villa méditerranéenne aux lignes géométriques, probablement perçue comme plus moderne.

\section{La position périphérique des thermes de Marlioz}

À côté de l'établissement thermal national, Aix-les-Bains a vu la création et le développement d'une seconde structure thermale, les thermes de Marlioz. Dû à l'initiative privée d'un négociant de Madrid, Barthélémy Billet, cet équipement inauguré en 1861 s'installe sur l'ancien domaine du château de Marlioz situé au sud-est de la ville (voir fig. 3). Alors que l'établissement national est spécialisé dans le traitement des rhumatismes, les thermes de Marlioz se consacrent aux soins des 
affections oto-rhino-laryngologiques. À l'exception du corps central, l'ensemble du bâtiment a été démoli et reconstruit en 1981. Les deux ailes latérales se prolongent par des galeries couvertes menant aux hôtels, permettant une circulation abritée entre les différents espaces. Les thermes de Marlioz ont cependant eu, et ont toujours, une position périphérique dans l'activité thermale de la ville. Cette place secondaire s'explique par leur implantation géographique, aux limites de la cité, ainsi que par le nombre de baigneurs accueillis, inférieur à celui du "géant» que représentait l'établissement thermal national.

24 À la fin du XIX et au début du Xx $x^{e}$ siècle, le secteur de Marlioz devient progressivement le lieu d'élection des infrastructures sportives. Alors que le tir aux pigeons, le golf et le stand de tir étaient disséminés dans la ville, les équipements tels que le nouveau golf (1905) et les tennis (1912) s'installent dans cette zone, à proximité et à la suite de l'ouverture de l'hippodrome (1884).

\section{Des fondations médicales issues du thermalisme}

$25 \mathrm{Au}$ cours des $\mathrm{XIX}^{\mathrm{e}}$ et $\mathrm{XX}^{\mathrm{e}}$ siècles, l'activité thermale suscite la création d'autres établissements médicaux prodiguant des soins présentés comme complémentaires de la cure thermale. Le corps médical aixois, par l'intermédiaire de la société médicale d'Aixles-Bains, ainsi que les baigneurs et les visiteurs étrangers, sont les moteurs de ces implantations. Les premiers légitiment le discours médical et constituent un vivier de personnel soignant tandis que les seconds représentent une clientèle potentielle ainsi qu'une source de financement important. À l'instar de l'hôpital thermal Reine Hortense, créé en 1816 par Mme Mère et le cardinal Fesch, et de l'institut de mécanothérapie Zander ouvert en 1899, bénéficiant de nombreux dons de la communauté étrangère, la construction de l'hôpital municipal et de l'hospice Brachet, inaugurés en 1907, est en grande partie financée par la colonie anglophone et plus particulièrement par l'industriel américain John Pierpont Morgan, qui séjourne à Aix vingt années durant ${ }^{10}$.

Parmi ces fondations médicales, la création du solarium tournant en 1930 retient l'attention par son originalité. Le principe curatif et le programme architectural de la station héliothérapique orientable ont été pensés par le docteur Jean Saidman, directeur de l'institut d'Actinothérapie. Cette branche de la médecine, constituée comme science dans les années 1920, développe des traitements curatifs reposant sur l'utilisation des rayonnements naturels du soleil et des rayonnements artificiels. Le solarium tournant, implanté sur les hauteurs de la ville afin de bénéficier de l'ensoleillement maximum (voir fig. 4), est doté de cabines de soins abritées au sein d'une "plateforme mobile » capable de suivre la course du soleil ${ }^{11}$ (fig. 15). De très beaux témoignages de cette construction étonnante, démolie dans les années 1960 tout comme le solarium de Vallauris dans les Alpes-Maritimes ${ }^{12}$, sont conservés grâce aux clichés réalisés par le photographe hongrois André Kertesz à l'occasion d'un reportage en Savoie en $1933^{13}$. 


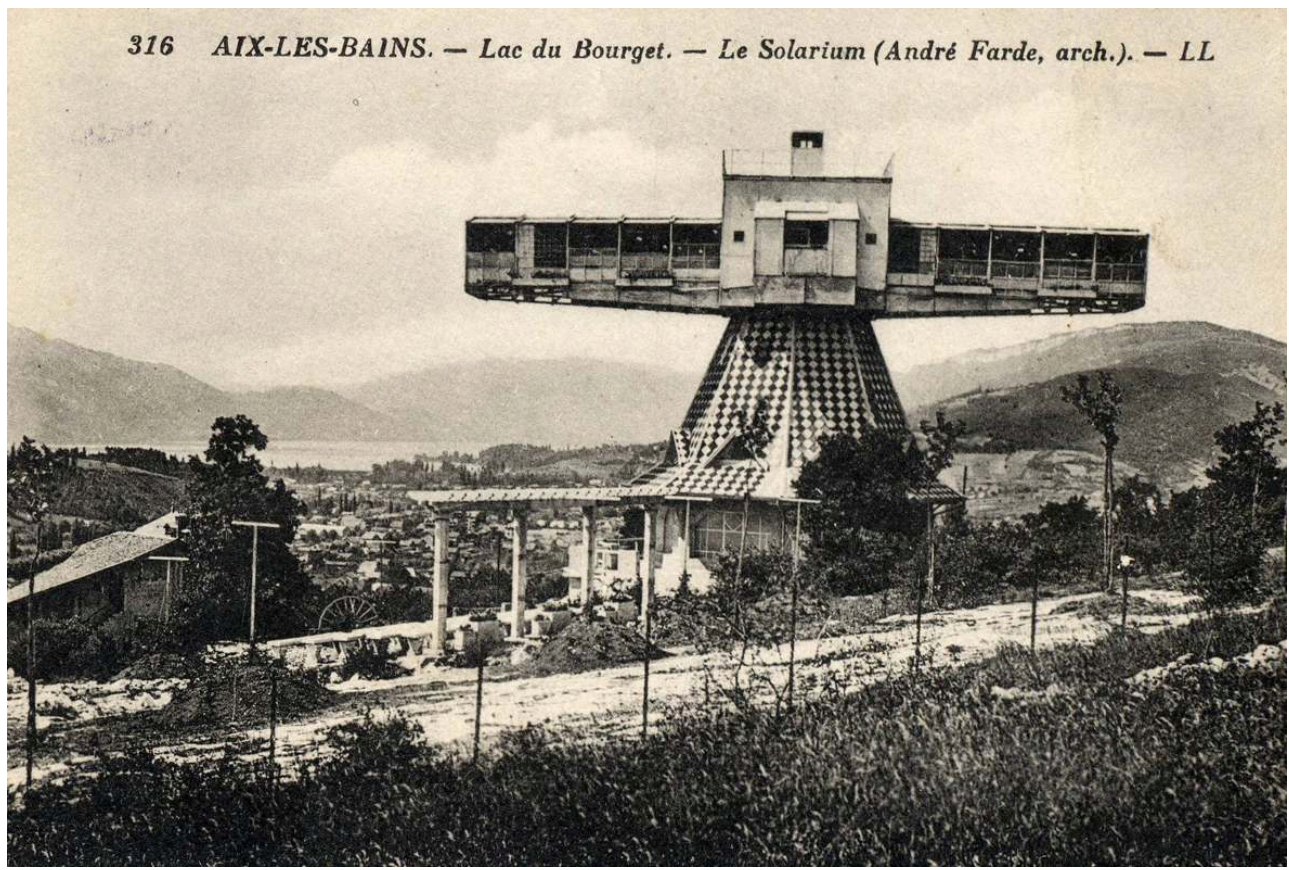

Le solarium tournant, années 1930.

(C) Archives municipales d'Aix-les-Bains.

\section{La conquête et le développement du Revard : du climatisme aux sports d'hiver ${ }^{14}$}

Dans la seconde moitié du XIX ${ }^{\mathrm{e}}$ siècle, l'intérêt pour la montagne et pour le Revard se développe chez les membres du Club Alpin Français (CAF), présent à Aix, chez les villégiateurs attirés de manière plus générale par la nature environnante et chez certains médecins aixois relayant le discours médical autour de la climatologie et du climatisme.

\section{Le rêve climatérique du docteur Jean Monard}

Surnommé "le père du Revard", le docteur Jean Monard, membre de la société médicale d'Aix et du CAF, est l'un des grands promoteurs du Revard. Nourri par les développements de la climatologie médicale, il effectue une visite des stations climatiques suisses et rhénanes et entreprend l'étude des climats par des mesures et des observations météorologiques. Fort de ces différentes expériences, il développe en 1890 le concept de "vallée climatérique Aix-Revard" s'articulant autour de quatre zones. Les bords de lac, le centre urbain, les balcons du Revard et le Revard constituent un ensemble reposant sur la complémentarité de ces quatre espaces disposant chacun de leurs caractéristiques climatiques propres ${ }^{15}$. La création de stations climatiques sur les balcons du Revard et sur le Revard est alors perçue comme un moyen supplémentaire pour attirer et surtout retenir la clientèle :

Pendant mon séjour à Falkenstein, je vis un malade pour qui la cure d'altitude était impérieusement exigée et il dut partir pour Davos, puisqu'il n'existe pas de station similaire en Allemagne, soit 24 heures de chemin de fer. On recule souvent devant 
la fatigue et les ennuis d'un pareil voyage. Chez nous, au contraire, on se transportera dans une demi-heure au plus d'une altitude à l'autre. Jamais nous ne garderons dans un climat qui serait mal toléré des malades, pour lesquels il nous sera toujours aisé de trouver la formule qui convient à leurs cas spéciaux ${ }^{16}$.

Si l'aménagement des thermes nationaux procède des modèles d'Europe de l'Est, celui de la montagne s'inspire directement des pratiques suisses. La Suisse semble en effet être une source d'inspiration, tant par sa proximité que par l'idée selon laquelle sa configuration géographique, caractérisée par des villes situées entre lacs et montagnes, est proche de celle d'Aix-les-Bains.

\section{L'aménagement climatique et touristique de la montagne}

Figure 16

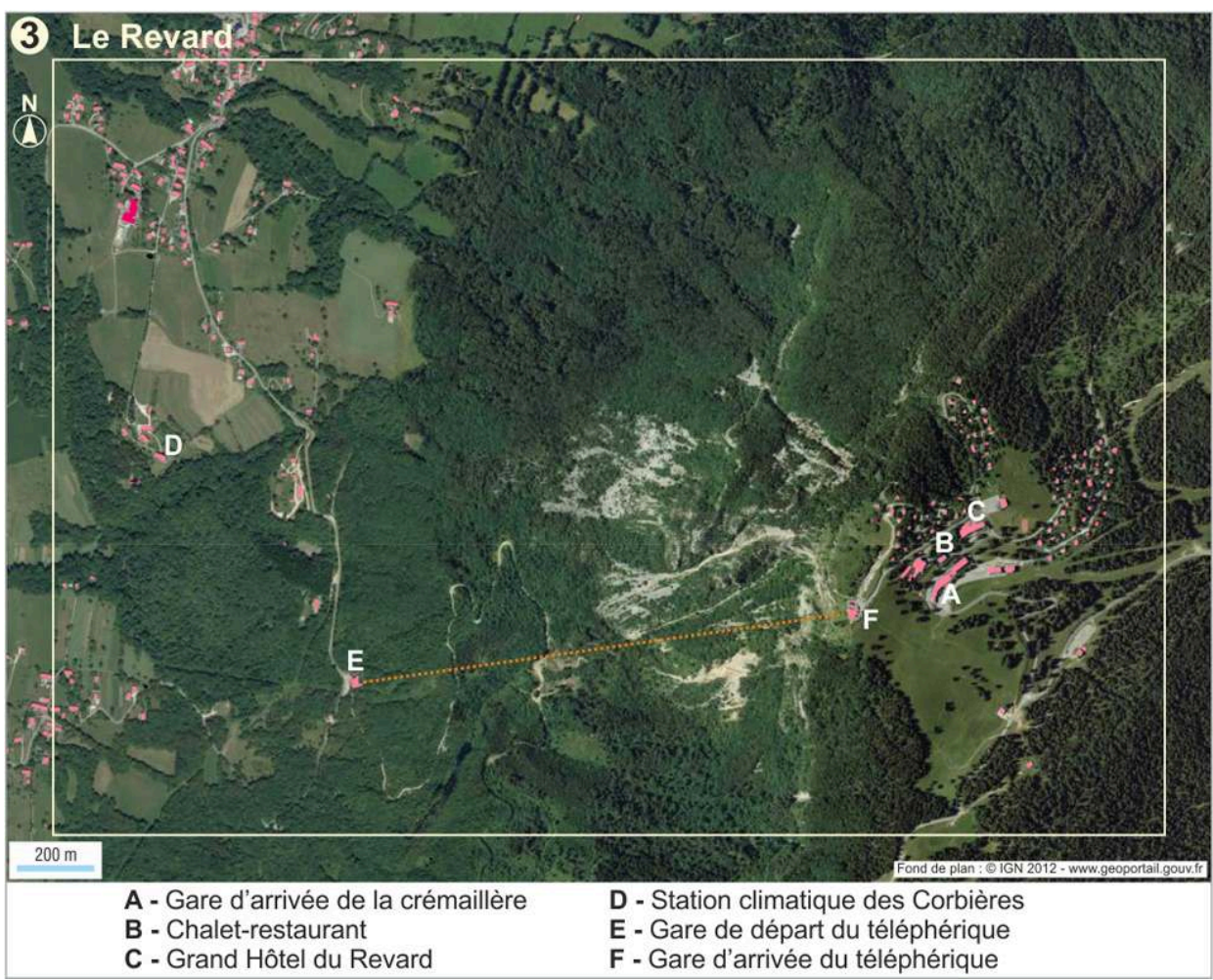

Carte de localisation, le Revard et ses balcons.

Cartog. Céréza, André, 2014. ( Région Rhône-Alpes, service de l'Inventaire général du patrimoine culturel. (c) Ville d'Aix-les-Bains.

\section{(fig. 16)}

31 La question de l'accessibilité du plateau du Revard mobilise les membres du CAF au cours du dernier quart du xix siècle. Elle trouve une solution en 1892 avec l'ouverture d'un chemin de fer à crémaillère reliant le centre-ville d'Aix-les-Bains au Revard et exploité par une société composée d'administrateurs et d'ingénieurs suisses ${ }^{17}$.

La gare de départ, le dépôt et les infrastructures de maintenance, aujourd'hui disparus, étaient situés au sud-est du parc thermal, à l'emplacement actuel du palais des Congrès (voir fig. 4). La gare se présentait comme un petit chalet dont la construction et le décor recouraient au bois. Deux des trois gares intermédiaires, ainsi que la gare d'arrivée située sur le plateau, revêtaient le même aspect. Dès l'inauguration du chemin 
de fer à crémaillère, un chalet-restaurant en 1892, puis deux chalets-hôtels en 1893-1894, ouvrent leurs portes sur le plateau du Revard. Ces constructions s'implantent à quelques mètres en amont de la gare d'arrivée et en contrebas du sommet, balayé par les vents, sur lequel seuls une station d'observation météorologique et un belvédère s'installent. Adossés à la pente, le chalet-restaurant et les deux chaletshôtels privilégient une orientation vers le sud-est, c'est-à-dire vers le soleil et vers le panorama des montagnes, aux dépens de la vue sur le lac visible depuis le sommet (voir fig. 16). Le Revard est alors fréquenté à la fois de manière journalière par des étrangers venus d'Aix-les-Bains en excursion, et de manière plus prolongée par des baigneurs et des convalescents auxquels les médecins recommandent le «bon air » après la cure (fig. 17). La station d'été du Revard est créée ex-nihilo.

Figure 17

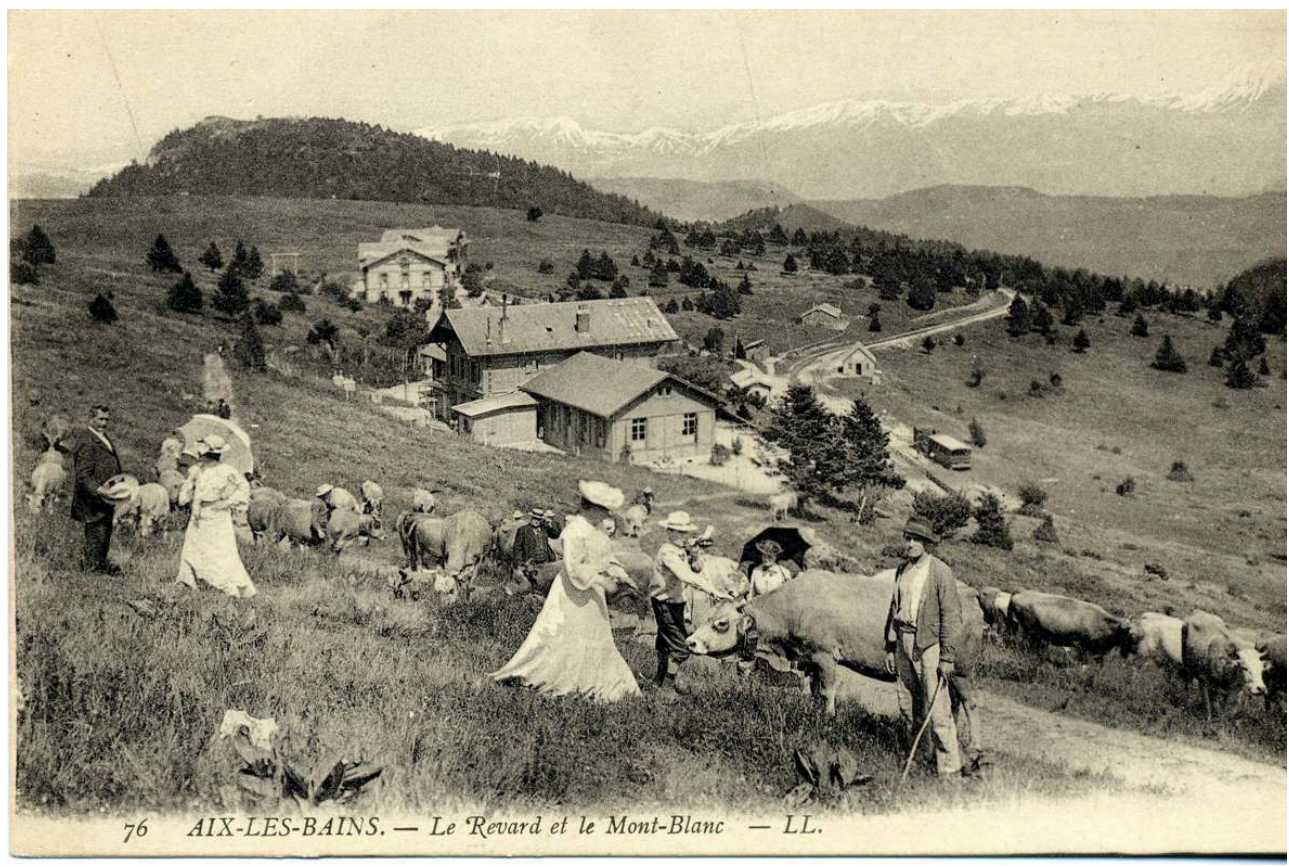

La station d'été du Revard, début du xxe siècle.

(c) Archives municipales d'Aix-les-Bains.

Parallèlement à l'aménagement du Revard, une société constituée notamment de Jean Monard, de Pierre-Victor Barbier, membre du CAF, et de Léon Grosse, fondateur de l'importante entreprise aixoise de bâtiments et travaux publics éponyme, est à l'origine de la création de la station climatique de moyenne altitude des Corbières (voir fig. 16) ${ }^{18}$. Implantée à 600 mètres d'altitude, elle est desservie par la gare intermédiaire de Pugny-Châtenod et inaugurée dès 1892. Malgré le séjour des reines de Hollande en 1896, cette opération se révèle cependant un échec commercial. Alors que le projet prévoyait la construction d'un grand hôtel de luxe logé au sein d'un parc agrémenté de promenades savamment étudiées, seuls une laiterie et un hôtel de taille beaucoup plus modeste voient le jour. La station climatique des Corbières ferme en 1917 et l'ancien hôtel, qui en constitue aujourd'hui le seul témoignage, abrite désormais les sœurs de Bethléem. La façade principale de ce bâtiment, orientée vers le sud-est, s'orne de loggias en bois qui permettaient de prendre le soleil et de respirer le bénéfique air alpin (fig. 18). 


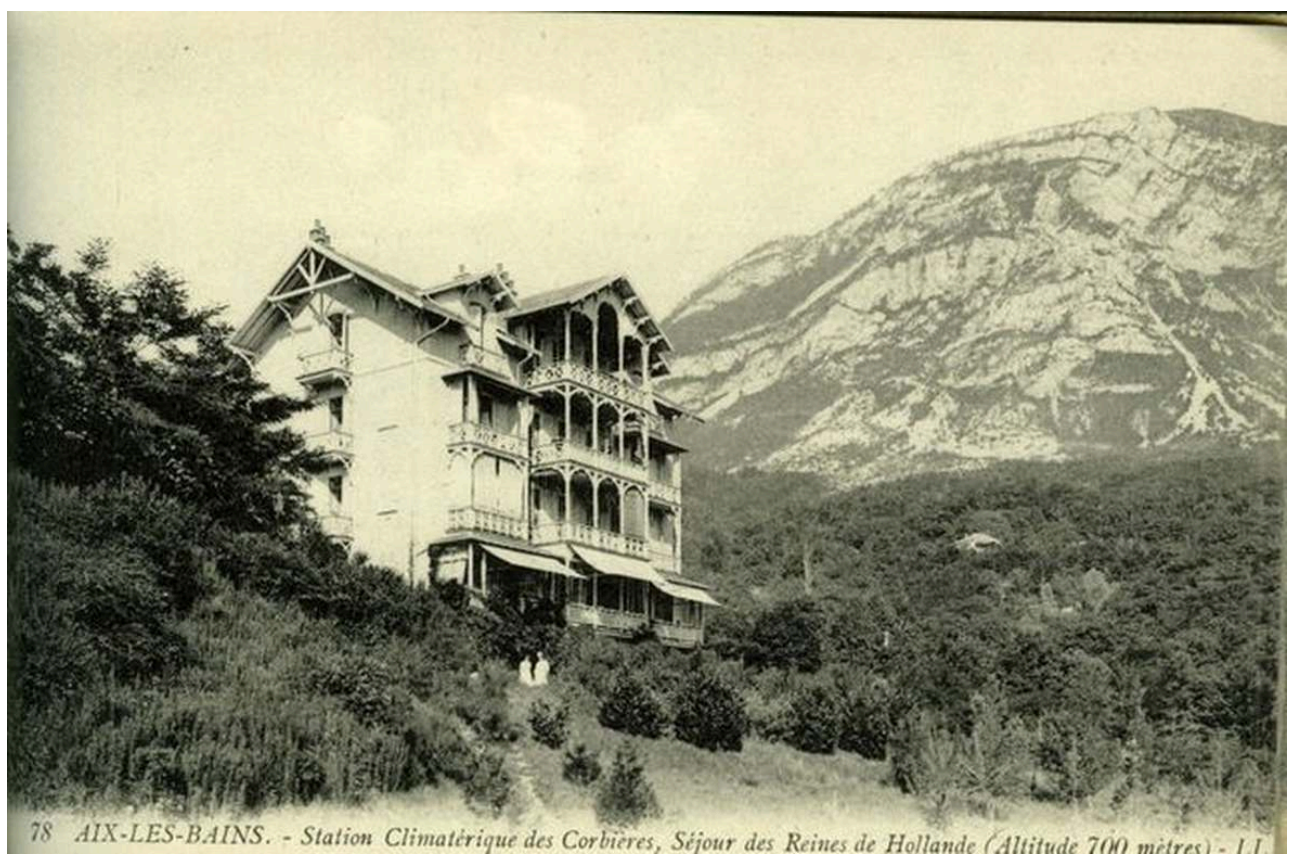

La station climatique des Corbières, début du $x x^{e}$ siècle.

(c) Archives municipales d'Aix-les-Bains

Dans la mesure où l'inauguration du chemin de fer à crémaillère, du chalet-restaurant et de la station climatique des Corbières ont lieu en 1892, il semble évident qu'accessibilité et aménagement de la montagne ont été pensés conjointement.

\section{La naissance et le développement des sports d'hiver au Revard}

Les liens entre le Revard et le thermalisme aixois se relâchent très progressivement à la suite des effets produits par deux faits marquants : le lancement de la saison d'hiver en 1909 et le rachat du domaine du Revard par la Compagnie Paris-Lyon-Méditerranée en 1923.

Jusqu'alors station d'été, la saison d'hiver au Revard est lancée en 1909 par la mise en place d'un service hivernal de trains et par une inauguration officielle. Au cours de celle-ci, l'organisation de concours de sports d'hiver avec des épreuves de glace, de luge, de bobsleigh, de saut à ski et de ski de fond, comme la présence du skieur norvégien Durban Hansen, démontrent la volonté de faire du Revard une véritable station d'hiver qui se concrétise par l'aménagement d'équipements permettant de pratiquer ces différents sports.

En 1923, la Compagnie Paris-Lyon-Méditerranée acquiert le domaine du Revard et entreprend des travaux visant à augmenter la capacité d'accueil de la station et à en améliorer la qualité. La mise en place d'un service de trains plus régulier s'accompagne de la reconstruction de la gare d'arrivée, confiée à l'architecte Pierre Patout ${ }^{19}$, ainsi que de la surélévation et de la transformation des deux chalets-hôtels et du chaletrestaurant menées par l'architecte annécien Fleury Raillon ${ }^{20}$ (fig. 19). 
Figure 19

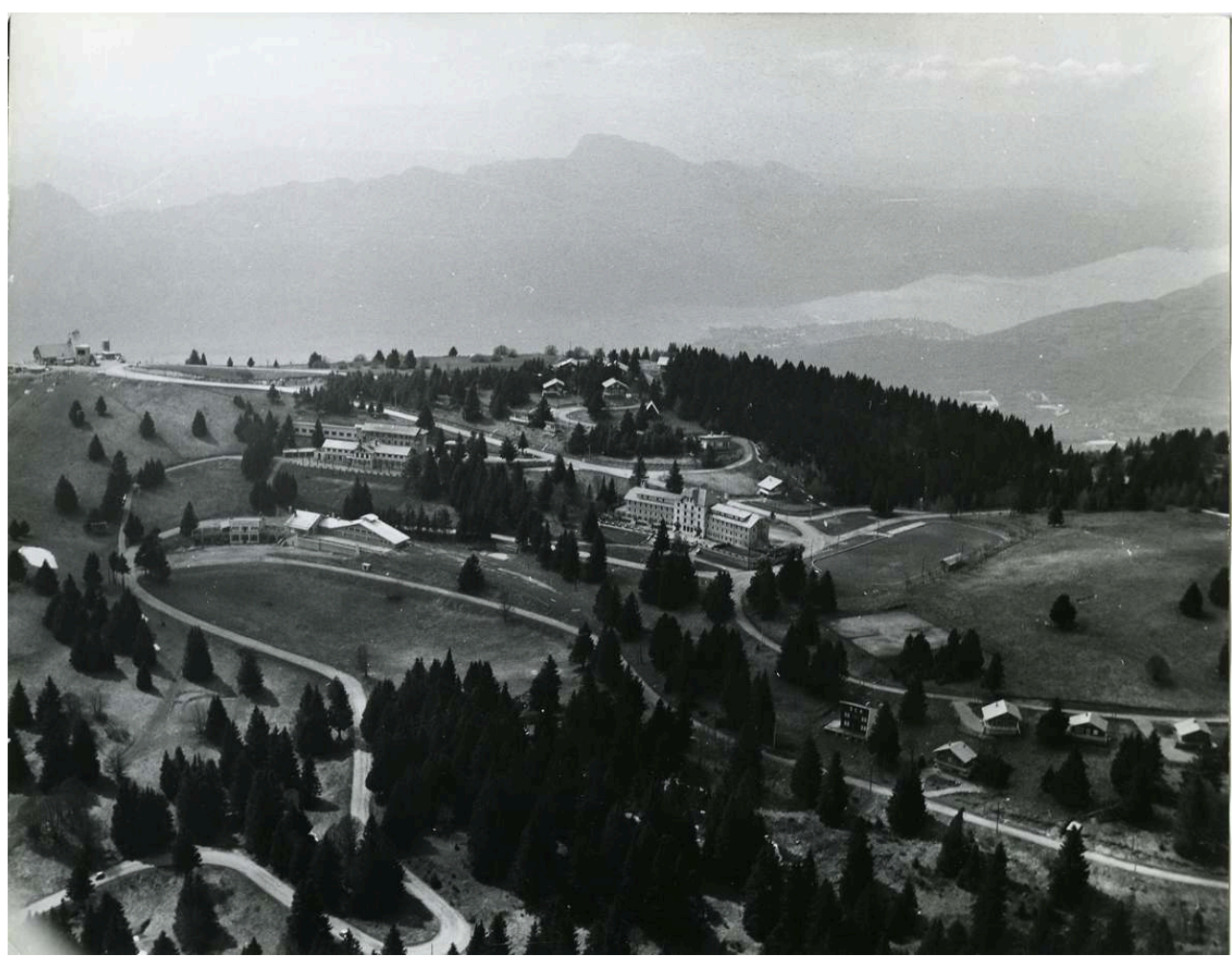

Vue aérienne du domaine du Revard, fin des années 1930.

(c) Archives municipales d'Aix-les-Bains.

À partir du milieu des années 1920, le Revard se dote d'équipements et d'infrastructures lui permettant de s'affranchir de la clientèle de l'activité thermale aixoise et de poursuivre une logique de développement plus autonome actée au milieu des années 1930. En 1932, l'orientation vers le développement des sports d'hiver au Revard se confirme par l'installation des premières remontées mécaniques. En 1935, la Compagnie Paris-Lyon-Méditerranée inaugure un téléphérique doté d'une gare d'arrivée située au sommet du Revard et d'une gare de départ ${ }^{21}$ (fig. 20) implantée sur la commune de Mouxy, distante de plusieurs kilomètres d'Aix-les-Bains (voir fig. 16). Le second tronçon, destiné à relier Mouxy au centre d'Aix, ne verra jamais le jour tandis que le service du chemin de fer à crémaillère cesse dès 1937 et que les infrastructures ferroviaires sont démontées presque immédiatement. 
Figure 20

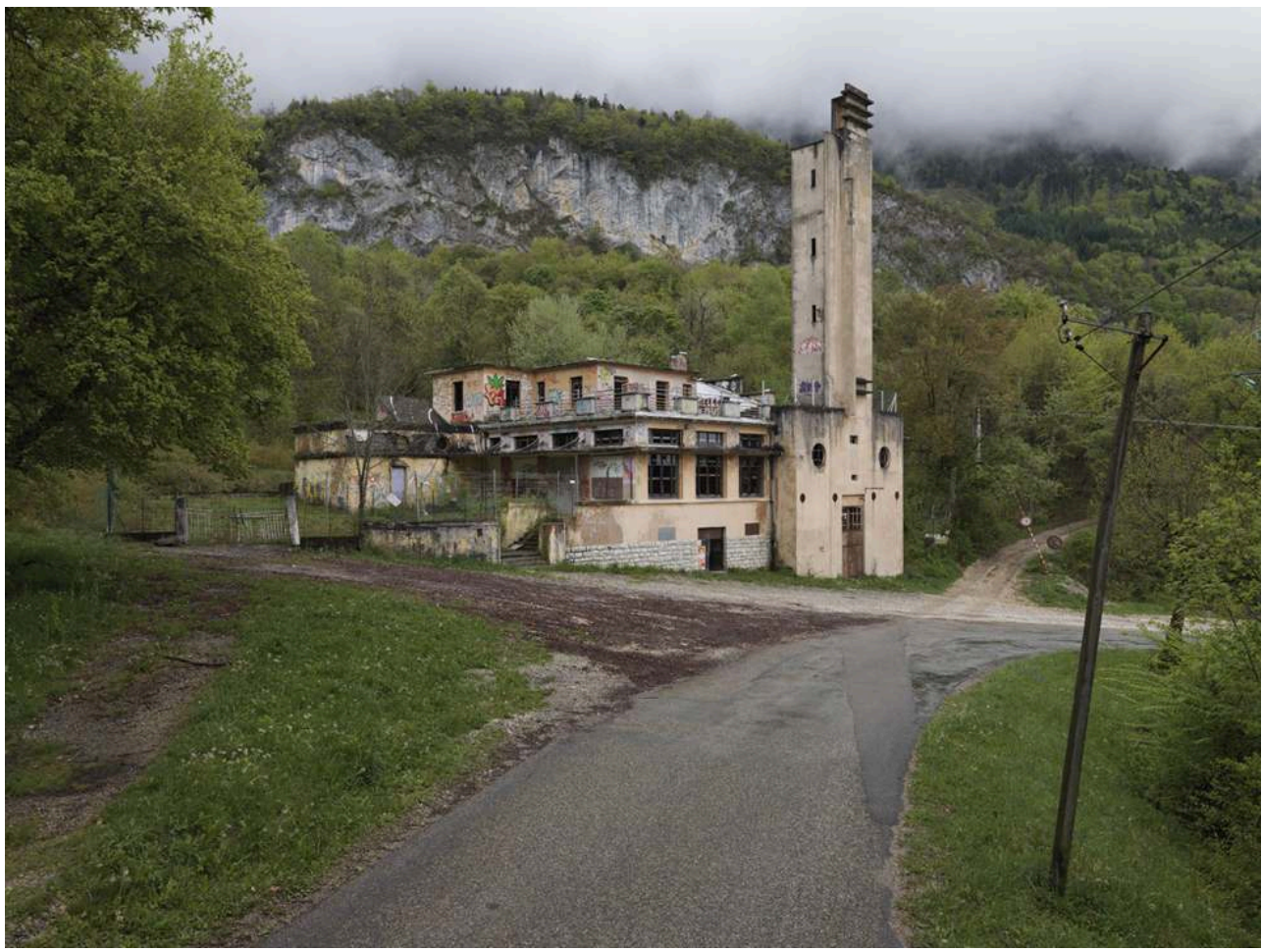

Gare de départ du téléphérique du PLM, à Mouxy.

Phot. Dessert, Éric, 2014. @ Région Rhône-Alpes, service de l'Inventaire général du patrimoine culturel. (c) Ville d'Aix-les-Bains.

39 Vendu et morcelé en 1953, le domaine du Revard est resté préservé puisque les différents projets d'aménagement et d'urbanisation dont il a fait l'objet par la suite n'ont jamais été réalisés. L'architecture des gares d'arrivée de la crémaillère et du téléphérique, le chalet-restaurant et les deux chalets-hôtels n'ont pas été dénaturés et l'organisation des bâtiments les uns par rapport aux autres reste très lisible.

\section{La progressive et lente appropriation des bords de lac}


Figure 21

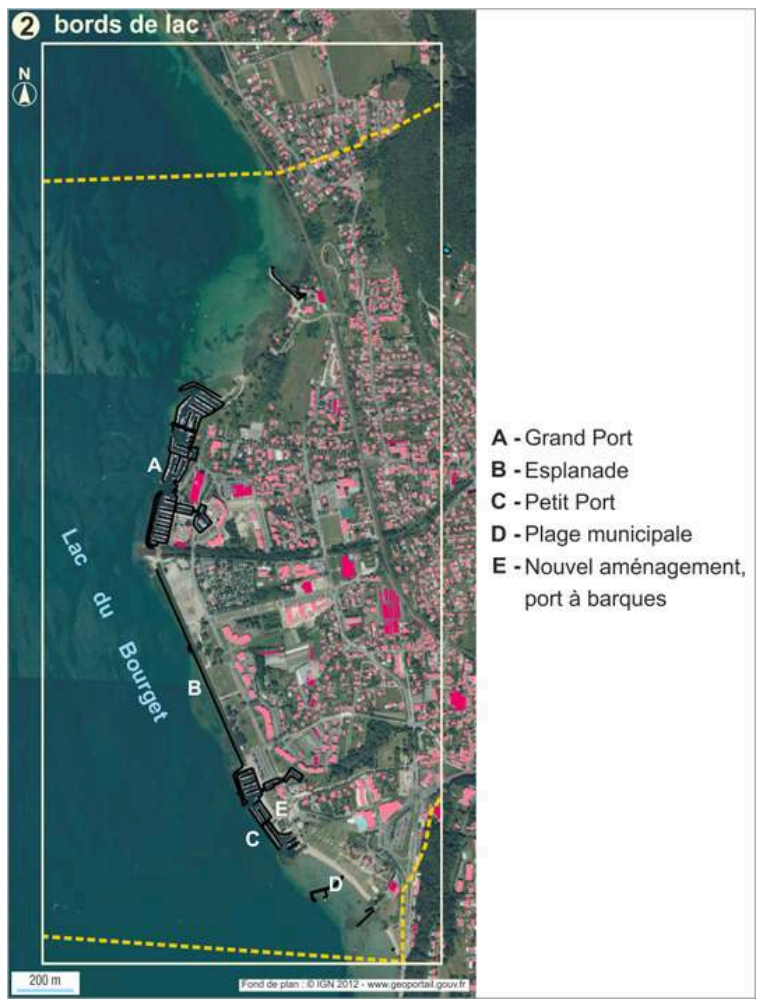

Carte de localisation, les bords de lac.

Cartog. Céréza, André, 2014. ( ) Région Rhône-Alpes, service de l'Inventaire général du patrimoine culturel. (c) Ville d'Aix-les-Bains.

Les bords de lac sont depuis longtemps utilisés pour le commerce des marchandises pouvant transiter jusqu'au Rhône par l'intermédiaire du canal naturel de Savière situé au nord. À l'inverse du Revard, le lac et ses abords ne sont exploités comme lieu de plaisance et de villégiature que progressivement et tardivement. En effet, les bords de lac sont pendant longtemps des zones de marais régulièrement inondées. Par ailleurs, si l'imaginaire collectif se représente la ville d'Aix bordée par les eaux du lac, c'est que la publicité y a grandement contribué. Or, le centre historique de la ville, regroupé autour de l'établissement thermal, se trouve en réalité relativement éloigné du lac.

\section{L'aménagement des ports}

$42 \mathrm{Au}$ cours des $\mathrm{XIX}^{\mathrm{e}}$ et $\mathrm{XX}^{\mathrm{e}}$ siècles, deux ports sont progressivement aménagés. Ils permettent dans un premier temps le transport des marchandises, puis celui des personnes, avant d'accueillir des bateaux d'excursion et de plaisance.

L'aménagement du Grand Port, situé au nord, débute en 1784 par l'aménagement d'un môle à l'initiative du gouvernement sarde. L'apparition des premiers bateaux à vapeur, en 1839, coïncide avec les premières excursions organisées pour les baigneurs et touristes. Un second port, appelé Petit Port car de taille plus modeste, est créé en 1886 par la Ville à l'embouchure du Tillet, au sud du Grand Port (voir fig. 21). À la fin du XIX siècle, les villégiateurs fréquentent quotidiennement les bords de lac, pour de simples promenades ou des excursions en bateau (fig. 22). Grand Port et Petit Port deviennent 
facilement accessibles par le service de tramways reliant le centre historique, actif entre 1896 et $1908^{22}$. Mais si ces deux ports deviennent des lieux de plaisance, ils ne deviennent pas pour autant de véritables lieux de villégiature s'incarnant dans la présence de villas.

Figure 22

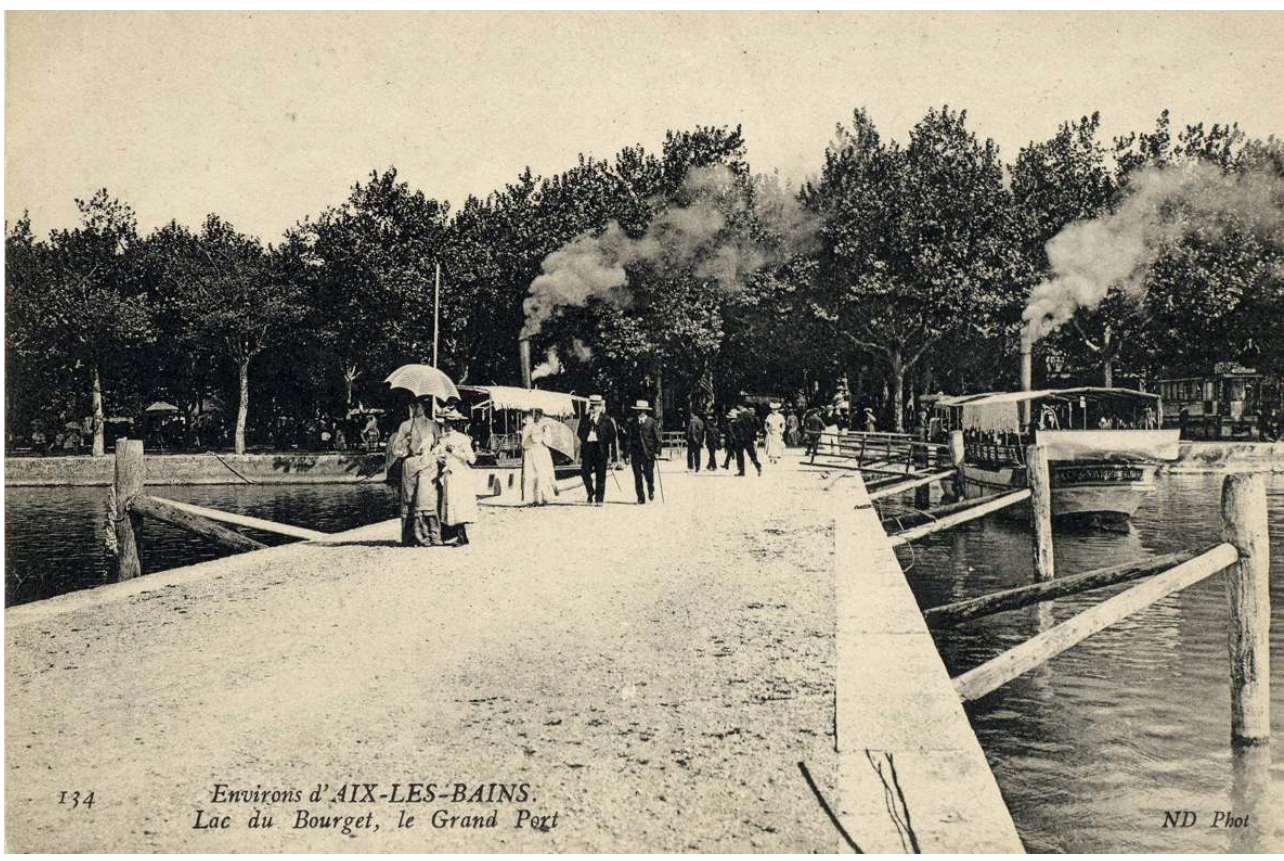

Déambulations sur le Grand Port, début du xxe siècle.

(c) Archives municipales d'Aix-les-Bains.

À partir du début du siècle, ce sont les axes reliant le lac au centre historique, et plus particulièrement l'avenue du Grand-Port, qu'investissent les villas. Ces constructions, plus modestes que celles bâties sur les Coteaux, ont été bien préservées, comme cette villa édifiée en 1903 pour un rentier parisien au sein d'un vaste jardin (fig. 23). Cependant, l'avenue du Grand-Port comme les zones proches des bords de lac ont été également conquises à partir des années 1930 par les résidences principales, souvent occupées par des retraités. Dans ce secteur de la ville et à l'image de l'île-de-France, l'étude de l'habitation pose ainsi la question des frontières entre les notions de villégiature et d'habitat pavillonnaire ainsi que celle d'un possible élargissement de la première à la situation des retraités ${ }^{23}$. 


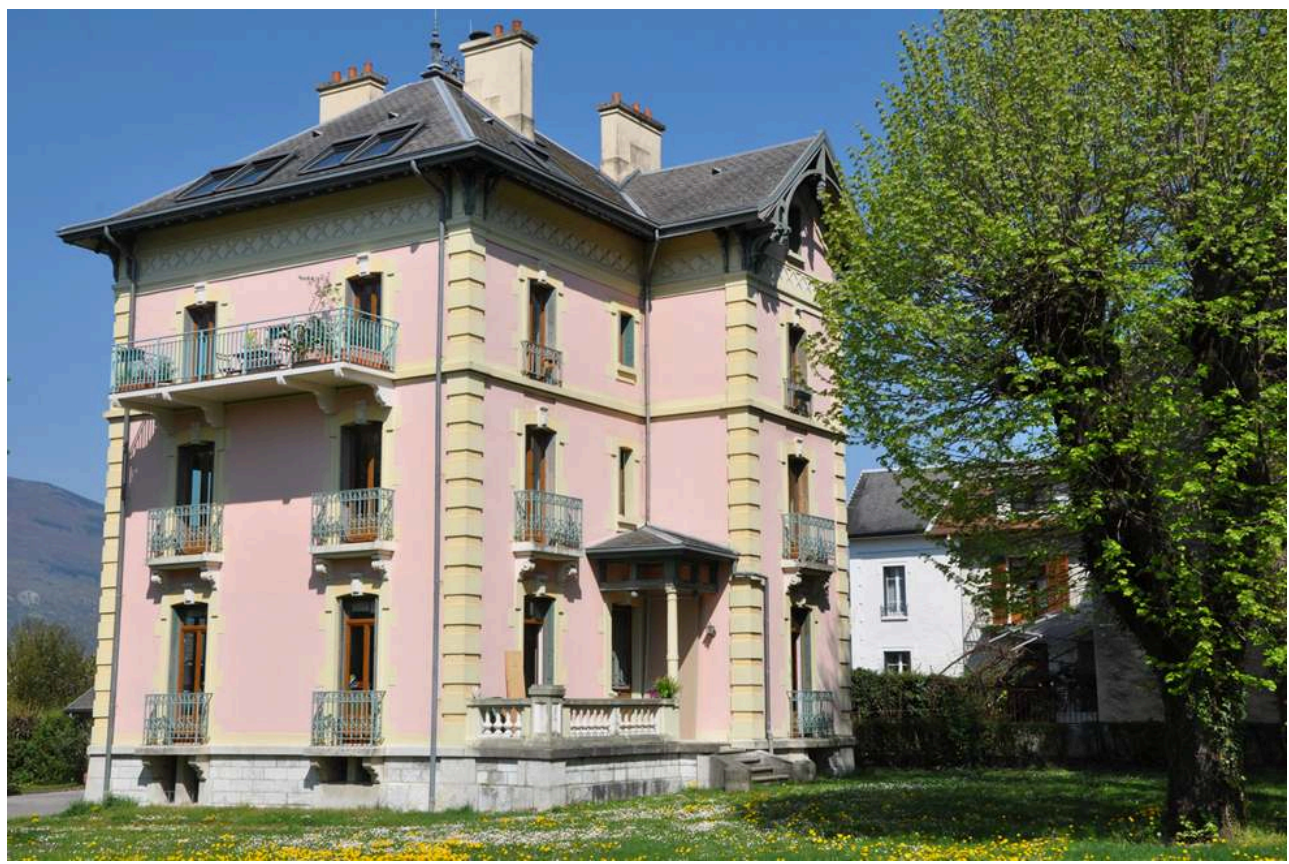

Villa sur l'avenue du Grand Port.

Phot. Harreau, Denys, 2013. @ Région Rhône-Alpes, service de l'Inventaire général du patrimoine culturel. (c) Ville d'Aix-les-Bains.

\section{La plage municipale d'Aix-les-Bains}

Outre le réaménagement du parc thermal, les travaux menés sur l'établissement thermal au début des années 1930 occasionnent la création d'une plage municipale au bord du lac par la Ville qui confie le chantier à l'architecte Roger Pétriaux. Une telle initiative puise son inspiration en Suisse, référence explicitement mentionnée dans les délibérations du conseil municipal.

Implantée au sud du Petit Port, au lieu-dit Coëtan, la plage s'installe sur des marais, ce qui nécessite d'importants travaux de remblayage, et forme une anse orientée vers le sud-ouest (voir fig. 21). Cet aménagement dans le style Art déco, où l'on reconnaît le geste de Roger Pétriaux, est pensé et conçu comme un équipement luxueux, ce qui se traduit par le choix de matériaux et de mobilier de qualité, l'adoption d'une politique tarifaire sélective et l'organisation d'animations régulières. La plage adopte un plan symétrique organisé autour d'un axe central formé par une abside. Occupée par un restaurant, celle-ci est couverte d'une terrasse utilisée comme solarium. Les vestiaires sont logés dans quatre absides implantées de part et d'autre. Deux douches en forme de champignon prennent place entre les vestiaires et le rivage. Depuis la rue, l'entrée de la plage est signalée par une tour encadrée de deux portiques reliés à deux corps de bâtiment rectangulaire (fig. 24). Excepté la mise en place de grilles permettant de guider la circulation, la plage municipale est très bien conservée et aujourd'hui encore, très fréquentée durant la saison estivale. 
Figure 24

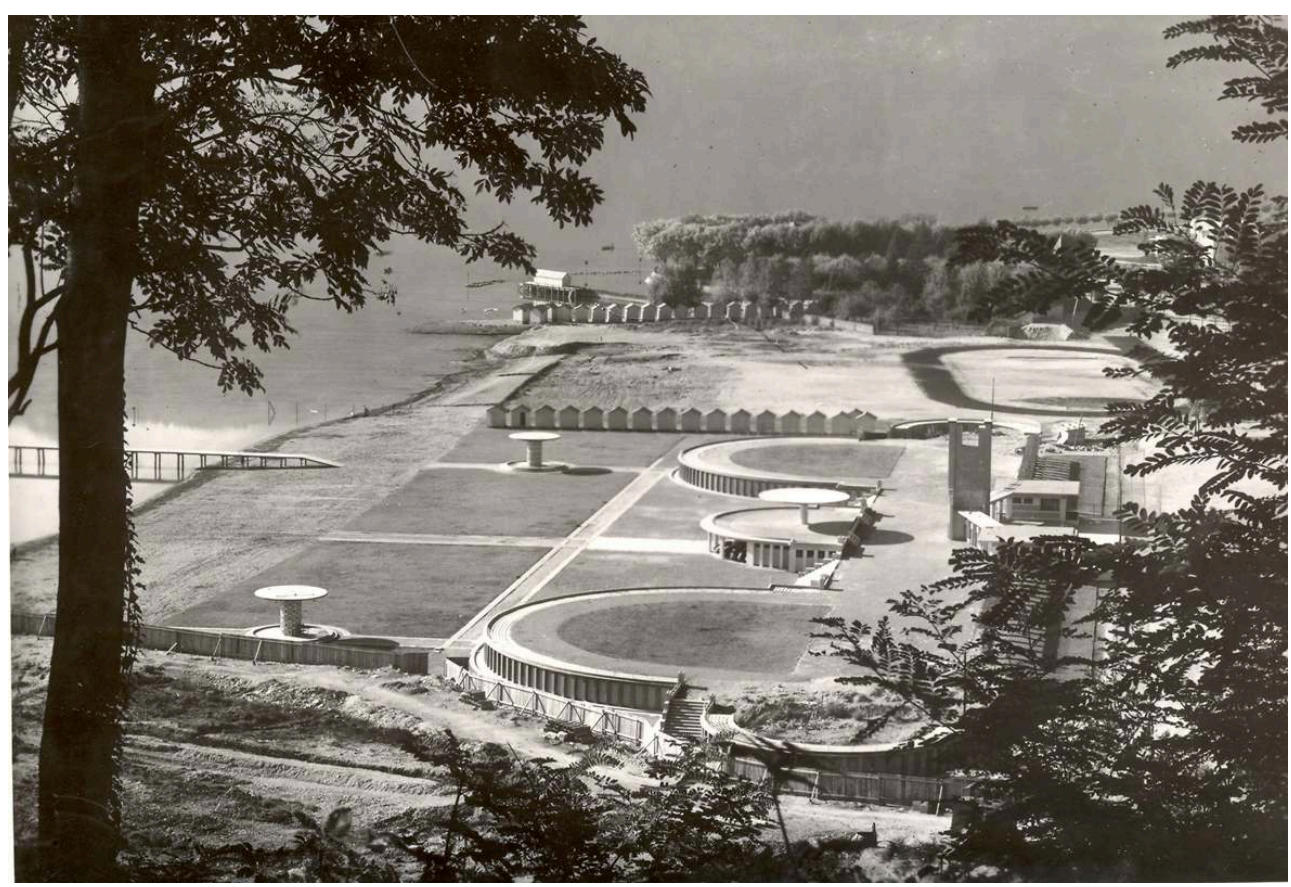

Vue aérienne de la plage municipale d'Aix, années 1930

(c) Archives municipales d'Aix-les-Bains

\section{Un processus à l'œuvre}

Depuis les années 1930, les bords de lac font l'objet d'un intérêt constant et croissant de la part des aménageurs publics et privés. Dans la seconde moitié $\mathrm{du} \mathrm{xx}^{\mathrm{e}}$ siècle, la création d'une promenade, signalée par deux rangées de platanes, permet de relier le Petit Port et le Grand Port (voir fig. 21). Celle-ci est prolongée d'une grande esplanade offrant la possibilité d'accueillir différents événements tels que le festival de musique Musilac, depuis 2002. Jusqu'ici peu prisés, les bords de lac ont été bâtis : un front de lac, constitué d'hôtels, d'immeubles de logements et de bureaux, a été construit à quelques dizaines de mètres du rivage, comprenant également un camping (fig. 25). Aujourd'hui, les bords de lac sont l'objet de nombreux projets d'aménagement urbain destinés à en faciliter l'accès pour la déambulation et à en accroître l'attractivité immobilière. Un nouveau port à barques, dont les abords ont été réorganisés pour accueillir la construction d'immeubles, a été inauguré par la municipalité au début de l'année 2014 (voir fig. 21).

\section{Conclusion}

Désormais, l'attention des pouvoirs publics et privés se concentre en priorité sur les bords de lac et la représentation de la ville d'Aix-les-Bains dans l'imaginaire collectif est presque exclusivement liée au lac du Bourget. Pourtant, le thermalisme n'en reste pas moins un domaine clef.

49 Bien que les palaces et les grands hôtels aient fermé tour à tour leurs portes depuis les années 1950, le tissu hôtelier, et plus généralement, l'activité commerciale demeurent 
importants dans l'économie aixoise et reposent en grande partie sur la présence des curistes. Or, le thermalisme a connu récemment d'importants bouleversements. En effet, l'implantation séculaire de l'établissement thermal et sa centralité dans la ville ont été remises en question par la construction d'un nouveau bâtiment de bains situé sur les hauteurs. Initiée par l'État, l'érection des thermes Chevalley a été confiée à l'architecte Stanislas Fiszer et menée entre 2000 et 2005 (voir fig. 4). Par ailleurs, le thermalisme aixois, nationalisé depuis 1860 à la demande de Napoléon III, a été privatisé entre 2010 et 2012 : les thermes Chevalley, l'appellation Thermes nationaux ainsi que le parc thermal ont été vendus à la société privée Valvital, qui est à la tête d'une douzaine de stations thermales dans toute la France. L'établissement thermal historique, désaffecté depuis le transfert de l'intégralité des soins dans les thermes Chevalley en 2008, a quant à lui été vendu à la Ville. Actuellement, le bâtiment n'est que très partiellement occupé par les services municipaux, l'office de tourisme et une école d'esthétique. La grande majorité de l'espace, fermée au public, s'est transformée en friche et la municipalité est confrontée à la reconversion de 50000 mètres carrés de planchers.

Face à ces récents bouleversements, l'équipe d'Inventaire chargée de l'étude d'Aix-lesBains a réalisé une visite virtuelle à $360^{\circ}$ des anciens Thermes nationaux afin de restituer ces lieux et de les rendre accessibles via une interface numérique. Une étude des savoir-faire est apparue également nécessaire à la Société d'Art et d'Histoire d'Aixles-Bains, commanditaire d'une enquête ethnologique sur les techniques thermales aixoises, réalisée par Juliette Roland ${ }^{24}$. Celle-ci soulignait à juste titre le fait que l'histoire médicale thermale à Aix-les-Bains restait à faire.

Figure 25

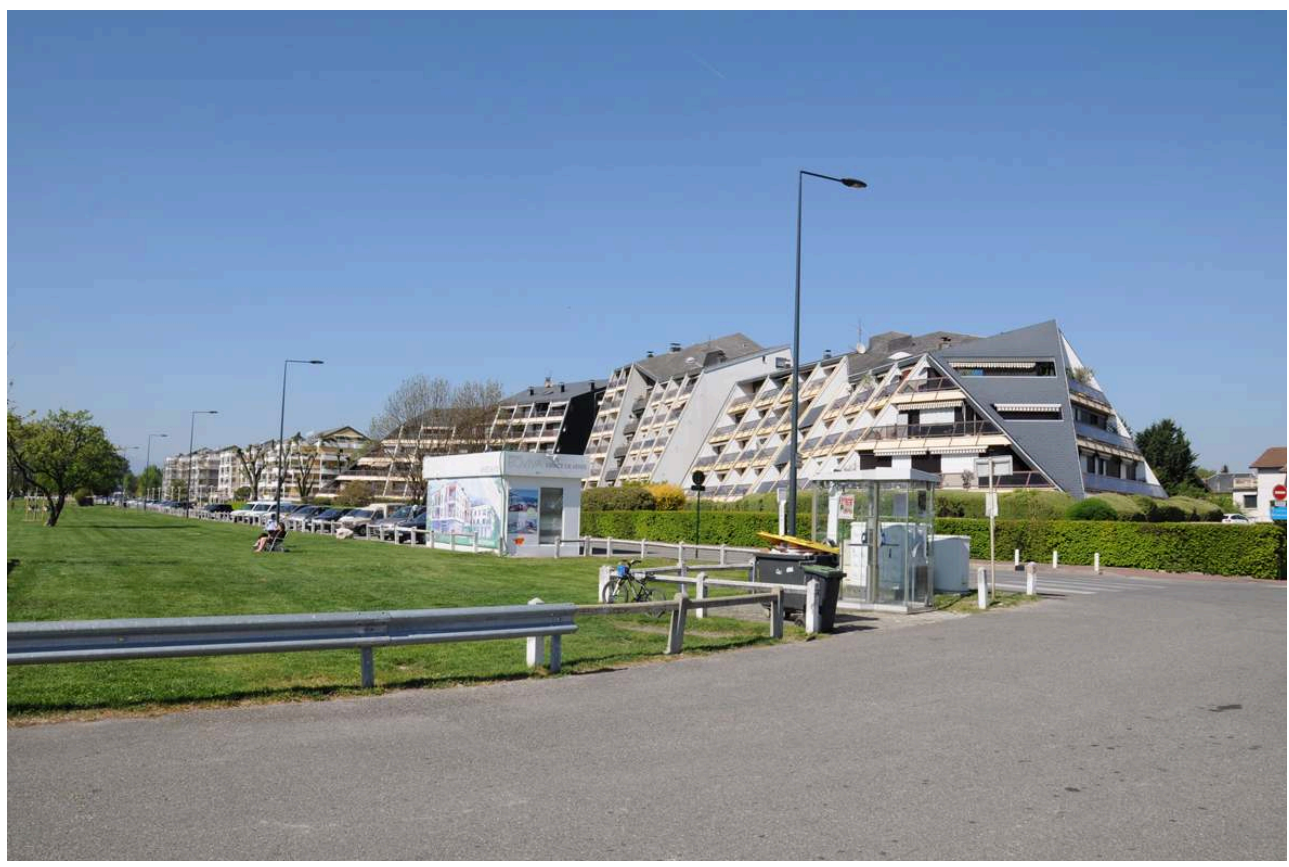

L'esplanade du lac bordée d'immeubles.

Phot. Harreau, Denys, 2014. @ Région Rhône-Alpes, service de l'Inventaire général du patrimoine culturel. (c) Ville d'Aix-les-Bains. 


\section{NOTES}

1. - Voir aussi : BELLE, Elsa. « Aix-les-Bains, carrefour de villégiatures : thermalisme, climatisme, sports d'hiver et bords de lac ». Les Carnets de l'Inventaire : études sur le patrimoine - Région Rhône-Alpes [en ligne], 16 juillet 2014.

2. - Site Internet de la Ville d'Aix-les-Bains. Voir le site : http://www.aixlesbains.fr/.

3. - Le terme de «station mondaine » est notamment employé dans les délibérations du conseil municipal, la presse et les guides touristiques.

4. - Le terme "baigneurs » renvoie plus précisément aux personnes fréquentant l'établissement thermal, et dont la désignation actuelle est celle de curistes ; le terme « étrangers », que l'on peut assimiler au terme contemporain de "touristes", désigne plus spécifiquement l'entourage accompagnant la personne suivant la cure ainsi que les autres personnes en villégiature, non originaires d'Aix-les-Bains et fréquentant la station de manière discontinue. Baigneurs et étrangers, curistes et touristes, sont des villégiateurs.

5. - LAMARTINE, Alphonse de. «Le lac ». Méditations poétiques, 1820.

6. - GRAS, Philippe, JAZÉ-CHARVOLIN, Marie-Reine. Aix en 1960. Aix-les-Bains : Société d'Art et d'Histoire, 2010 (coll. Arts et Mémoire, n59).p. 50.

7. - Cette façade a été redécouverte à l'occasion de la rénovation de l'établissement thermal menée par l'architecte Stanislas Fiszer en 2005.

8. - MOUXY DE LOCHE, François de. Histoire d'Aix-les-Bains. Chambéry : Imprimerie Savoisienne, 1898 , p. 363.

9. - L'hôtel Astoria, construit au début du $\mathrm{xx}^{\mathrm{e}}$ siècle, est également un hôtel à atrium.

10. - PAGE, Léon. Promenons nous dans les rues d'Aix. Aix-les-Bains : ville d'Aix, 1978, p. 202. L'hôpital municipal fait par ailleurs l'objet de recherches en cours.

11. - LEFEBVRE, Thierry, RAYNAL, Cécile. Les Solariums tournants du Dr Jean Saidman. Aix-les-Bains, Jamnagar, Vallauris. Paris : Éditions Glyphe, 2010.

12. - Trois solariums tournants ont été construits dans le monde : le premier à Aix-les-Bains, le deuxième à Vallauris et le troisième à Janmagar en Inde, le seul subsistant aujourd'hui.

13. - Les photographies réalisées par André Kertesz sont accessibles dans la base Mémoire du ministère de la Culture.

14. - Concernant la création et le développement du Revard, voir : Le Revard. Aix-les-Bains : Société d'Art et d'Histoire, 1996 (coll. Arts et Mémoire, nº).

15. - L'Avenir d'Aix-les-Bains, 7 décembre 1890, n71. L'Avenir d'Aix-les-Bains, 14 décembre 1890, $\mathrm{n}^{\circ} 72$.

16. - L'Avenir d'Aix-les-Bains, 7 décembre $1890, \mathrm{n}^{\circ} 71$.

17. - FOUGER, François. "L'importance des sociétés de chemin de fer dans le développement de la première station de ski française, le Mont-Revard ». Les réseaux de la villégiature, In Situ, revue des patrimoines [document électronique]. Paris: Ministère de la Culture, 2004: http:// insitu.revues.org/1906 [consulté le 04/07/2014].

18. - CONNILLE, Jean-François. Les Corbières, Histoire et spiritualité. Aix-les-Bains : Société d'Art et d'Histoire, 2005.

19. - Les plans de la gare du chemin de fer à crémaillère dressés par Pierre Patout sont conservés à la Cité de l'Architecture et du Patrimoine et accessibles depuis Internet : voir le site http:// archiwebture.citechaillot.fr/fonds/FRAPN02_PATPI/inventaire.

20. - Un corps central permettant de relier les deux chalets-restaurants est construit en 1897. En 1923, celui-ci est exhaussé de trois étages et l'ensemble du bâtiment surélevé d'un étage. Le chalet-restaurant est quant à lui doté d'un étage supplémentaire. 
21. - L'activité de la gare de téléphérique de Mouxy cesse en 1968. Aujourd'hui propriété de la Communauté d'agglomération du Lac du Bourget (CALB), ce bâtiment vétuste et désaffecté est promis à la démolition faute de projet de reconversion.

22. - FOUGER, François. Les Tramways d'Aix-les-Bains. Aix-les-Bains : Société d'Art et d'Histoire, 1997 (coll. Arts et Mémoire, n9).

23. - Dès les années 1930 et jusqu'à aujourd'hui, un nombre important de personnes choisit de s'installer dans cette ville pour la retraite.

24. - ROLAND, Juliette. L'Histoire des techniques thermales à Aix-les-Bains. Aix-les-Bains : Société

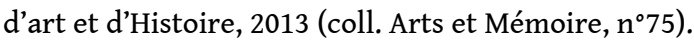

\section{RÉSUMÉS}

Située entre le lac du Bourget et le massif des Bauges, et implantée sur des sources d'eau chaude, la ville d'Aix-les-Bains a connu un formidable essor lié à l'activité thermale. Le thermalisme a suscité le développement du climatisme puis des sports d'hiver au Revard dans les Bauges ainsi que celui des activités balnéaires sur les rives du lac. Cette situation géographique et cette configuration historique ont fait d'Aix-les-Bains un carrefour de villégiatures unique en France dont l'héritage est plus que prégnant aujourd'hui.

Aix-les-Bains is a city located (settled) on thermal springs and situated between the lake of Bourget and the massif des Bauges mountains. This city knew an important development thanks to the spa facilities. Climatism, then winter sports in Revard and seaside activities on the lake's shores have been developed due to the influence of balneology. This geographic situation added to this historical construction created a forum of architectures which is the heritage of the city.

\section{INDEX}

Mots-clés : Rhône-Alpes, Savoie, Aix-les-Bains, villégiature, thermalisme, station thermale, station mondaine, sports d'hiver, climatisme, station climatique, activités balnéaires, lac du Bourget, montagne, massif des Bauges, mont Revard, établissement thermal, thermes romains, Thermes nationaux, thermes Chevalley, institut Zander, John Pierpont Morgan, solarium tournant, Jean Saidman, château de la Roche-du-Roi, architecture, urbanisme, chemin de fer à crémaillère, plage, casino, Charles Pellegrini, Roger Pétriaux, Claude Mabileau, parc thermal, Compagnie des chemins de fer Paris-Lyon-Méditerranée, curistes, baigneurs, Jean Monard, Samuel Revel, Bouhana, Lamartine, Napoléon III, palace, villa, Mouxy, gare de téléphérique, Pierre Patout, Fleury Raillon, Antoine Gouy, Rossignoli, Alfred Olivet, Jules Pin, thermes de Marlioz, « bon air », Corbières, Art déco, Edgard Brandt, Gentil et Bourdet, Stanislas Fiszer, visite virtuelle 
AUTEUR

ELSA BELLE

chercheure, Région Rhône-Alpes, service de l'Inventaire général du patrimoine culturel ebelle@rhonealpes.fr 